OPEN ACCESS

Edited by:

Giuseppe Mandaglio, University of Messina, Italy

Reviewed by: Egle Tomasi,

CEA Saclay, France Rishi Sharma,

Tata Institute of Fundamental Research, India

*Correspondence:

Fu-Hu Liu

fuhuliu@163.com fuhuliu@sxu.edu.cn

Khusniddin K. Olimov khkolimov@gmail.com kh.olimov@uzsci.net

Specialty section: This article was submitted to Nuclear Physics, a section of the journal Frontiers in Physics

Received: 09 October 2021 Accepted: 08 November 2021 Published: 17 December 2021

Citation:

Wang Q, Liu F-H and Olimov KK (2021) Initial-State Temperature of Light Meson Emission Source From Squared Momentum Transfer Spectra

in High-Energy Collisions.

Front. Phys. 9:792039.

doi: 10.3389/fphy.2021.792039

\section{Initial-State Temperature of Light Meson Emission Source From Squared Momentum Transfer Spectra in High-Energy Collisions}

\author{
Qi Wang ${ }^{1}$, Fu-Hu Liu ${ }^{1 *}$ and Khusniddin K. Olimov ${ }^{2 *}$ \\ ${ }^{1}$ State Key Laboratory of Quantum Optics and Quantum Optics Devices and Collaborative Innovation Center of Extreme Optics, \\ Institute of Theoretical Physics, Shanxi University, Taiyuan, China, ${ }^{2}$ Laboratory of High Energy Physics, Physical-Technical \\ Institute of Uzbekistan Academy of Sciences, Tashkent, Uzbekistan
}

The squared momentum transfer spectra of light mesons, $\pi^{0}, \pi^{+}, \eta$, and $\rho^{0}$, produced in high-energy virtual photon-proton $\left(\gamma^{*} p\right) \rightarrow$ meson + nucleon process in electron-proton (ep) collisions measured by the CLAS Collaboration are analyzed by the Monte Carlo calculations, where the transfer undergoes from the incident $\gamma^{\star}$ to emitted meson or equivalently from the target proton to emitted nucleon. In the calculations, the Erlang distribution from a multi-source thermal model is used to describe the transverse momentum spectra of emitted particles. Our results show that the average transverse momentum $\left(\left\langle p_{T}\right\rangle\right)$ and the initial-state temperature $\left(T_{i}\right)$ increase from lower squared photon virtuality $\left(Q^{2}\right)$ and Bjorken variable $\left(x_{B}\right)$ to higher one. This renders that the excitation degree of emission source, which is described by $\left\langle p_{T}\right\rangle$ and $T_{i}$, increases with increasing of $Q^{2}$ and $x_{B}$.

Keywords: initial-state temperature, average transverse momentum, squared momentum transfer, Erlang distribution, multi-source thermal model

\section{INTRODUCTION}

In the evolution process of high-energy nucleus-nucleus (heavy-ion) collisions, the reaction system undergoes several main stages which are separately the incoming of nuclei, beginning of collisions, strongly-coupled quark-gluon plasma (sQGP) phase or hot-dense matter phase, mixed phase, and hadron gas. In the stage of the incoming of nuclei, two nuclei move toward each other in vacuum tunnel at nearly the speed of light and change the shape to pancake with the Lorentz contraction. The sQGP phase is extremely hot-dense matter and the system can be regarded as a fireball. Considering the effect of pressure gradient, the system begins to inflate and cool down. Then, the hadron matter appears until the system is hadronic. To understand the mechanism of nuclear reaction and the property of system evolution, it is necessary to investigate the characteristics of each stage of collision process. The excitation and equilibrium degrees of the system are among very important characteristics [1-10].

To describe the excitation degree of the system, various temperatures of the system and the average transverse momentum $\left(\left\langle p_{T}\right\rangle\right)$ of particles are used [11-18]. The various temperatures include, but are not limited to, 1$)$ the initial-state temperature $\left(T_{i}\right)$ which reflects the temperature in the beginning of collisions of two nuclei, 2) the chemical freeze-out temperature $\left(T_{c h}\right)$ which reflects the temperature at chemical freeze-out when inelastic collisions disappear, 3 ) the kinetic freeze-out or final-state temperature $\left(T_{k i n}\right.$ or $\left.T_{0}\right)$ which reflects the temperature at kinetic freeze-out when 
elastic collisions disappear, and 4$)$ the effective temperature $\left(T_{\text {eff }}\right)$ which is not a "real" temperature, in which the influence of flow effect is not excluded compared with $T_{k i n}$ or $T_{0}$. Different kinds of temperatures can be "measured" by different "thermometers" (methods).

As the earliest temperature in collisions, $T_{i}$ is used to explore the secret of high-energy collisions [11-15]. As we know, $T_{i}$ is the temperature of emission source or interacting system when the system undergoes the initial-stage of collisions [19]. It is interesting for us to describe the excitation degree of the system by using $T_{i}$. Generally, from the transverse momentum $\left(p_{T}\right)$ spectra or fitting the $p_{T}$ spectra with different distributions or functions, we may obtain $T_{i}$. The Erlang distribution [20-22], Tsallis distribution [23, 24], Hagedorn function [25] are usually used, but in this paper, we only choose the Erlang distribution due to its origin of multiple sources in the multi-source thermal model [20-22]. In the special case, such as absent $p_{T}$ spectra, the squared momentum transfer spectra are alternatively used. Obviously, $T_{i}$ can not be obtained from the squared momentum transfer spectra directly unless the $p_{T}$ spectra are transformed to them. From the fit to $p_{T}$ spectra, $\left\langle p_{T}\right\rangle$ can be naturally abstracted.

In the transformation of $p_{T}$ spectra to squared momentum transfer spectra [26], the Monte Carlo method is used. First of all, concrete $p_{T}$, satisfying the Erlang distribution [20-22], are produced. Then, the squared momentum transfers are calculated according to the relation between squared momentum transfers and $p_{T}$ by using the Monte Carlo method. At last, the distribution of squared momentum transfer spectra are obtained and used to fit the experimental data for extracting $\left\langle p_{T}\right\rangle$ and $T_{i}$.

To describe the equilibrium degree of the system, one can use the Tsallis distribution [23, 24] or Hagedorn function [25] to fit $p_{T}$ spectra directly. In the fitting process, the entropy index $q$ can be extracted. The closer to 1 the entropy index $q$ is, the higher the degree of equilibrium of the source or system is. The relation between the two distributions is that the former one covers the later one in which the mass is neglected. Because the universality, similarity, or common characteristics exist in high-energy collisions [27-36], some distributions used in large collision system can be also used in small collision system. Although the equilibrium degree is also important, it is not discussed in this work due to other topics being concerned. We think that the equilibrium degree is enough to use the concept of temperature.

Meson consists of a quark and anti-quark $(q \bar{q})$ and belongs to hadron. It takes part in the strong interaction and play an important role. Light meson refers to a kind of meson with low mass. The transverse momentum of light meson changes more sensitively than that of the heavy one. Therefore, the study of transverse momentum spectra of light mesons is very important to explore the reaction mechanism and evolution process of high-energy collisions.

Compared with large systems of high-energy nucleus-nucleus collisions, small systems such as high-energy electron-proton, proton-proton, proton-nucleus collisions also produce abundant results. In particular, in electron-proton collisions, the scattered electron exchanges virtual photon $\left(\gamma^{*}\right)$ with the target proton.
Then, one may study high-energy $\gamma^{*}$ induced proton collisions, that is $\gamma^{*} p$ collisions, experimentally, theoretically as well as phenomenologically.

In this paper, the squared momentum transfer spectra of light mesons, $\pi^{0}, \pi^{+}, \eta$, and $\rho^{0}$, produced in high-energy $\gamma^{*} p$ collisions measured by the CLAS Collaboration [37-40] are fitted by the results originating from the Erlang $p_{T}$ distribution with the Monte Carlo method. The CLAS experimental data are measured at different squared photon virtuality $Q^{2}$ and Bjorken variable $x_{B}$, where $Q^{2}$ and $x_{B}$ will be discussed later in the Subsection 2.3.

\section{FORMALISM AND METHOD}

\subsection{The Erlang Distribution}

The Erlang distribution is a direct result of the multi-source thermal model [20-22]. One or two-component Erlang distribution can describe the narrow or wide $p_{T}$ spectra of particles, where the narrow (wide) $p_{T}$ spectra refers to range less than a few $\mathrm{GeV} / c$ (more than $10 \mathrm{GeV} / c$ ) [22]. The multisource thermal model assumes that multiple sources are formed in high-energy collisions. These sources can be nucleons or partons if we study the formation of nucleon clusters (nuclear fragments) or particles.

In this work, we assume that a few $\left(n_{s}\right)$ partons (partons-like) contribute to $p_{T}$ of a given particle [22]. The contribution of the $j$ th parton is assumed to be an exponential function with variable $p_{t j}$ which depends on $j$, and average value $\left\langle p_{t}\right\rangle$ which is independent of $j$. We have the normalized exponential function

$$
f\left(p_{t j}\right)=\frac{1}{\left\langle p_{t}\right\rangle} \exp \left(-\frac{p_{t j}}{\left\langle p_{t}\right\rangle}\right) .
$$

Here, $\left\langle p_{t}\right\rangle$ represents the average contribution of participant partons to $\left\langle p_{T}\right\rangle$ of the considered particles.

The contribution sum $\left(p_{t 1}+p_{t 2}+\cdots+p_{t n_{s}}\right)$ of $n_{s}$ partons is $p_{T}$ of a given particle. The result convoluting the contributions of $n_{s}$ partons is the Erlang distribution. We have the Erlang $p_{T}$ distribution to be

$$
f\left(p_{T}\right)=\frac{1}{N} \frac{d N}{d p_{T}}=\frac{p_{T}^{n_{s}-1}}{\left(n_{s}-1\right) !\left\langle p_{t}\right\rangle^{n_{s}}} \exp \left(-\frac{p_{T}}{\left\langle p_{t}\right\rangle}\right) .
$$

Here $N$ is the number of particles, and the form of $(1 / N) d N / d p_{T}$ results in the normalization of $f\left(p_{T}\right)$ to 1 . In fact, the normalization of the Erlang distribution is naturally 1 .

We would like to emphasize here the difference between " $n_{s}$ ", the number of partons and " $N$ ", the number of particles. In $\gamma^{*} p$ collisions, if three quarks in the proton contributed to $p_{T}$, we have $n_{s}=3$. If another $q \bar{q}$ pair also contributed to $p_{T}$, we have $n_{s}=3+$ $2=5$. Even in nucleus-nucleus collisions, the value of $n_{s}$ is not large due to it being determined by the number of contributor partons in a nucleon-nucleon pair, but not collision system itself. This makes sense, in the Fock's first two terms of the development of the wave function of the proton, as composed by 3 quarks and then 3 quarks plus a $q \bar{q}$ pair $[41,42]$. As for $N$, its value may be small in small collision system or peripheral nucleus-nucleus 


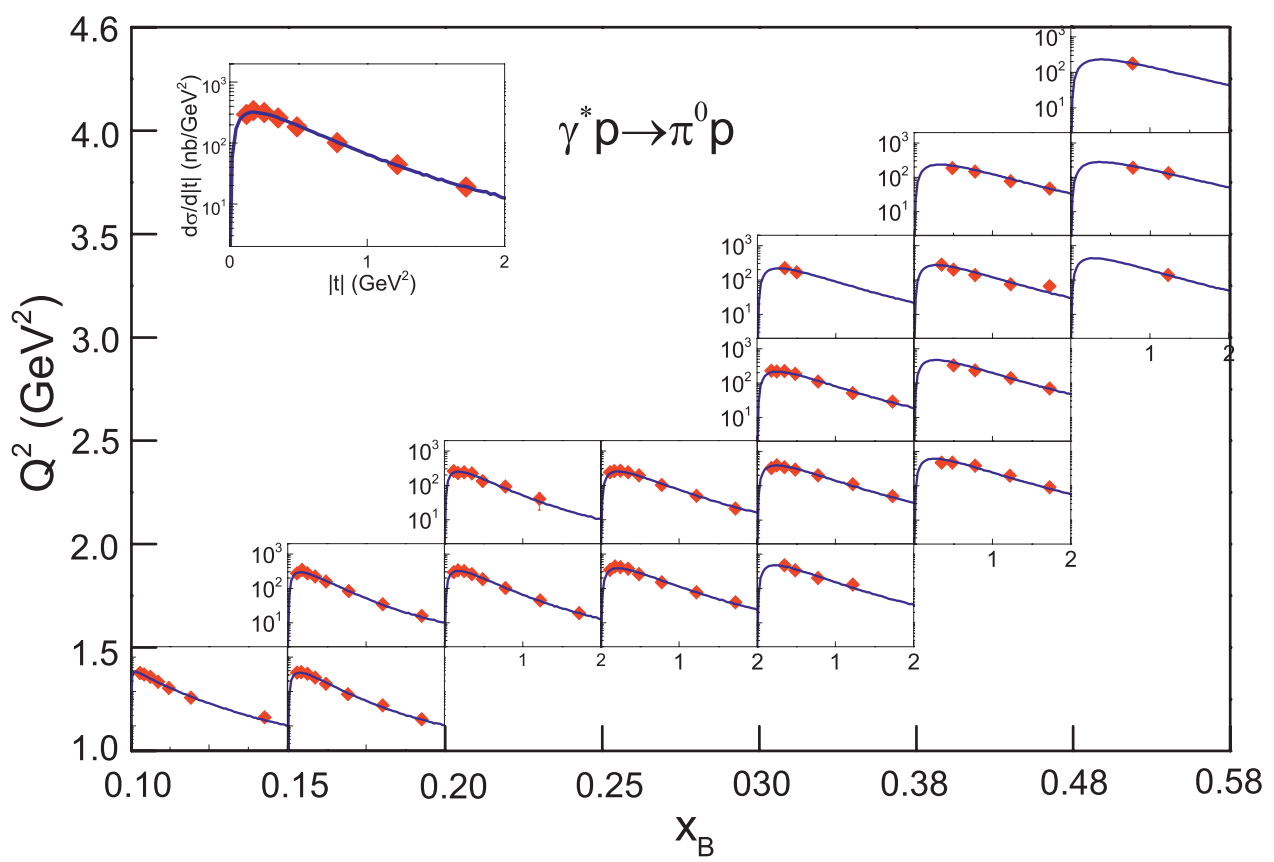

FIGURE 1 The differential cross-section $d \sigma / d|t|$ in $|t|$ of $\gamma^{\star} p \rightarrow \pi^{0} p$ process produced in ep collisions at beam energy of 5.75 GeV in different ranges of $Q^{2}$ and $x_{B}$ shown in the panels. The sample at the top-left sub-panel shows repeatedly the result in $1.5<Q^{2}<2.0 \mathrm{GeV}^{2}$ and $0.20<x_{B}<0.25$ as an example. The symbols represent the experimental data measured by the CLAS Collaboration [37] and the curves are the statistical results of $|t|\left(\right.$ Eq. 9) in which $p_{3 T}$ satisfies the Erlang distribution (Eq. 2) and can be obtained with the Monte Carlo method (Eq. 13).

collisions. The value of $N$ may be very large in central nucleusnucleus collisions at high energy.

\subsection{Average Transverse Momentum and Initial-State Temperature}

As we know, both the average transverse momentum $\left\langle p_{T}\right\rangle$ and initial-state temperature $T_{i}$ [11-15] describe the excitation degree of the system. In particular, in the Erlang distribution, $\left\langle p_{T}\right\rangle$ can be easily obtained by

$$
\left\langle p_{T}\right\rangle=\int_{0}^{\infty} p_{T} f\left(p_{T}\right) d p_{T}=n_{s}\left\langle p_{t}\right\rangle
$$

where $f\left(p_{T}\right)$ is normalized to 1 . Similarly, $\left\langle p_{t}\right\rangle$ reflects the excitation degree of participant partons.

According to Refs. [43-45], with a color string percolation method [46], $T_{i}$ can be regarded as

$$
T_{i}=\sqrt{\frac{\left\langle p_{T}^{2}\right\rangle}{2 F(\xi)}},
$$

where

$$
\left\langle p_{T}^{2}\right\rangle=\int_{0}^{\infty} p_{T}^{2} f\left(p_{T}\right) d p_{T}
$$

due to $f\left(p_{T}\right)$ is normalized to 1 and $\sqrt{\left\langle p_{T}^{2}\right\rangle}$ is the root-meansquare of $p_{T}$. In Eq. 4, $F(\xi)$ is the color suppression factor [46].

In the process of using color string method to obtain $T_{i}$ in this work, only one string is used, i.e., $F(\xi)=1$, in the formation of particle. Although there are probability to have any other strings, they do not affect noticeably $T_{i}$. If we consider other strings, according to Ref. [46], one has the minimum $F(\xi) \approx 0.6$. This will cause the maximum increase of $29.1 \%$ in $T_{i}$. Considering the fraction of one string is very large, that of two strings is relative small, and that of multiple strings is very small, the increase in $T_{i}$ will be much smaller than $29.1 \%$.

\subsection{The Squared Momentum Transfer}

In the center-of-mass reference frame, in two-body process $2+$ $1 \rightarrow 4+3$ or two-body-like process of high-energy collisions, there are three Mandelstam variables defined based on the fourmomenta of these particles. They have the forms to be

$$
\begin{gathered}
s=-\left(P_{1}+P_{2}\right)^{2}=-\left(P_{3}+P_{4}\right)^{2}, \\
t=-\left(P_{1}-P_{3}\right)^{2}=-\left(-P_{2}+P_{4}\right)^{2}, \\
u=-\left(P_{1}-P_{4}\right)^{2}=-\left(-P_{2}+P_{3}\right)^{2},
\end{gathered}
$$

where $P_{1}, P_{2}, P_{3}$, and $P_{4}$ are four-momenta of particles 1 (target proton), 2 (incident $\gamma^{*}$ ), 3 (emitted nucleon), and 4 (emitted meson), respectively. Here, we assume that particle 1 is incident along the $O z$ direction and particle 2 is incident along the opposite direction. After collisions, particle 3 is emitted with angle $\theta$ relative to the $O z$ direction and particle 4 is emitted along the opposite direction.

The three Mandelstam variables have different physical meaning. For instance, $\sqrt{s}$ refers to the center-of-mass energy, and $-u$ is defined as the squared momentum transfer between particles 1 and 4 . Here, selected variable $-t$ (the squared momentum transfer between particles 1 and 3) is calculated to fit the experimental data. For convenience, we have 
TABLE 1| Values of $E_{1},\left\langle p_{t}\right\rangle, n_{s}, \sigma_{0}, T_{i}$, and $\chi^{2} /$ ndof corresponding to the curves in Figures 1, 2, where $n_{s}$ is constrained to be integer with uncertainty of 0 which is not listed in the table. The number of parameters is always 4 which includes $E_{1},\left\langle p_{t}\right\rangle, n_{s}$, and $\sigma_{0}$. In the case of ndof being less than or equal to the number of parameters, we obtain the curve from a "prediction" or extrapolation based on other reasonable fits, and show the corresponding nop in a bracket to replace ndof. The value of $\chi^{2}$ is rounded to an integer, or one significant digit if the integer is 0 .

\begin{tabular}{|c|c|c|c|c|c|c|c|c|}
\hline Collisions & $\begin{array}{c}Q^{2} \\
(\mathrm{GeV})\end{array}$ & $x_{B}$ & $\begin{array}{c}E_{1} \\
(\mathrm{GeV})\end{array}$ & $\begin{array}{c}\left\langle p_{t}\right\rangle \\
(\mathrm{GeV} / c)\end{array}$ & $n_{s}$ & $\begin{array}{c}\sigma_{0} \\
(\mu \mathrm{b})\end{array}$ & $\begin{array}{c}T_{i} \\
(\mathrm{GeV})\end{array}$ & $\begin{array}{c}\chi^{2} / \text { ndof } \\
\text { (nop) }\end{array}$ \\
\hline \multirow[t]{18}{*}{$\gamma^{\star} p \rightarrow \pi^{0} p$} & $(1.0,1.5)$ & $(0.10,0.15)$ & $0.945_{-0.007}^{+0.015}$ & $0.176 \pm 0.002$ & 3 & $0.195 \pm 0.005$ & $0.409 \pm 0.005$ & $7 / 11$ \\
\hline & $(1.0,1.5)$ & $(0.15,0.20)$ & $0.945 \pm 0.004$ & $0.109 \pm 0.002$ & 5 & $0.220 \pm 0.008$ & $0.422 \pm 0.008$ & $11 / 12$ \\
\hline & $(1.5,2.0)$ & $(0.15,0.20)$ & $0.945 \pm 0.005$ & $0.113 \pm 0.002$ & 5 & $0.197 \pm 0.003$ & $0.438 \pm 0.008$ & $5 / 12$ \\
\hline & $(1.5,2.0)$ & $(0.20,0.25)$ & $0.945 \pm 0.003$ & $0.118 \pm 0.001$ & 5 & $0.232 \pm 0.010$ & $0.457 \pm 0.004$ & $6 / 12$ \\
\hline & $(2.0,2.5)$ & $(0.20,0.25)$ & $0.945_{-0.007}^{+0.015}$ & $0.118 \pm 0.004$ & 5 & $0.175 \pm 0.005$ & $0.457 \pm 0.015$ & $7 / 11$ \\
\hline & $(1.5,2.0)$ & $(0.25,0.30)$ & $0.945 \pm 0.001$ & $0.131 \pm 0.001$ & 5 & $0.335 \pm 0.013$ & $0.507 \pm 0.004$ & $12 / 12$ \\
\hline & $(2.0,2.5)$ & $(0.25,0.30)$ & $0.945 \pm 0.001$ & $0.131 \pm 0.001$ & 5 & $0.220 \pm 0.007$ & $0.507 \pm 0.004$ & $6 / 12$ \\
\hline & $(1.5,2.0)$ & $(0.30,0.38)$ & $0.945 \pm 0.002$ & $0.135 \pm 0.003$ & 5 & $0.430 \pm 0.020$ & $0.523 \pm 0.012$ & $7 /(4)$ \\
\hline & $(2.0,2.5)$ & $(0.30,0.38)$ & $0.945 \pm 0.003$ & $0.140 \pm 0.001$ & 5 & $0.380 \pm 0.013$ & $0.542 \pm 0.004$ & $10 / 11$ \\
\hline & $(2.5,3.0)$ & $(0.30,0.38)$ & $0.945 \pm 0.002$ & $0.142 \pm 0.001$ & 5 & $0.215 \pm 0.010$ & $0.550 \pm 0.004$ & $6 / 11$ \\
\hline & $(3.0,3.5)$ & $(0.30,0.38)$ & $0.945 \pm 0.003$ & $0.146 \pm 0.002$ & 5 & $0.230 \pm 0.011$ & $0.565 \pm 0.007$ & $0.8 /(2)$ \\
\hline & $(2.0,2.5)$ & $(0.38,0.48)$ & $0.945 \pm 0.005$ & $0.144 \pm 0.001$ & 5 & $0.630 \pm 0.030$ & $0.558 \pm 0.004$ & $14 / 9$ \\
\hline & $(2.5,3.0)$ & $(0.38,0.48)$ & $0.945 \pm 0.002$ & $0.147 \pm 0.002$ & 5 & $0.500 \pm 0.022$ & $0.569 \pm 0.007$ & $17 /(4)$ \\
\hline & $(3.0,3.5)$ & $(0.38,0.48)$ & $0.945 \pm 0.001$ & $0.150 \pm 0.002$ & 5 & $0.300 \pm 0.016$ & $0.581 \pm 0.008$ & 29/9 \\
\hline & $(3.5,4.0)$ & $(0.38,0.48)$ & $0.945 \pm 0.005$ & $0.160 \pm 0.003$ & 5 & $0.290 \pm 0.012$ & $0.620 \pm 0.012$ & $2 /(4)$ \\
\hline & $(3.0,3.5)$ & $(0.48,0.58)$ & $0.945 \pm 0.001$ & $0.151 \pm 0.002$ & 5 & $0.480 \pm 0.023$ & $0.585 \pm 0.008$ & $0.1 /(1)$ \\
\hline & $(3.5,4.0)$ & $(0.48,0.58)$ & $0.945 \pm 0.002$ & $0.170 \pm 0.003$ & 5 & $0.380 \pm 0.014$ & $0.658 \pm 0.012$ & $1 /(2)$ \\
\hline & $(4.0,4.6)$ & $(0.48,0.58)$ & $0.945 \pm 0.003$ & $0.172 \pm 0.005$ & 5 & $0.320 \pm 0.015$ & $0.666 \pm 0.020$ & $0.01 /(1)$ \\
\hline \multirow[t]{20}{*}{$\gamma^{\star} p \rightarrow \pi^{+} n$} & 1.75 & 0.25 & $0.950 \pm 0.005$ & $0.072 \pm 0.002$ & 5 & $1.200 \pm 0.050$ & $0.279 \pm 0.008$ & $18 / 13$ \\
\hline & 1.75 & 0.31 & $0.950 \pm 0.010$ & $0.075 \pm 0.004$ & 5 & $0.950 \pm 0.030$ & $0.290 \pm 0.015$ & $28 / 12$ \\
\hline & 2.05 & 0.25 & $0.950 \pm 0.010$ & $0.074 \pm 0.003$ & 5 & $0.650 \pm 0.020$ & $0.287 \pm 0.011$ & $16 / 11$ \\
\hline & 2.05 & 0.31 & $0.950 \pm 0.003$ & $0.076 \pm 0.006$ & 5 & $1.200 \pm 0.040$ & $0.294 \pm 0.023$ & $29 / 11$ \\
\hline & 2.05 & 0.37 & $0.950 \pm 0.010$ & $0.078 \pm 0.006$ & 5 & $2.400 \pm 0.090$ & $0.302 \pm 0.023$ & $27 / 11$ \\
\hline & 2.35 & 0.31 & $0.950 \pm 0.004$ & $0.078 \pm 0.010$ & 5 & $0.900 \pm 0.020$ & $0.302 \pm 0.039$ & $24 / 12$ \\
\hline & 2.35 & 0.37 & $0.950 \pm 0.010$ & $0.079 \pm 0.002$ & 5 & $2.500 \pm 0.080$ & $0.306 \pm 0.008$ & $9 / 11$ \\
\hline & 2.35 & 0.43 & $0.950 \pm 0.006$ & $0.110 \pm 0.010$ & 5 & $2.500 \pm 0.100$ & $0.426 \pm 0.039$ & $10 / 11$ \\
\hline & 2.65 & 0.31 & $0.950 \pm 0.010$ & $0.079 \pm 0.002$ & 5 & $0.900 \pm 0.030$ & $0.306 \pm 0.008$ & $16 / 11$ \\
\hline & 2.65 & 0.37 & $0.950 \pm 0.008$ & $0.083 \pm 0.003$ & 5 & $1.900 \pm 0.050$ & $0.321 \pm 0.012$ & $14 / 10$ \\
\hline & 2.65 & 0.43 & $0.950 \pm 0.010$ & $0.111 \pm 0.004$ & 5 & $2.000 \pm 0.070$ & $0.430 \pm 0.016$ & $9 / 10$ \\
\hline & 2.95 & 0.37 & $0.950 \pm 0.010$ & $0.111 \pm 0.010$ & 5 & $0.700 \pm 0.020$ & $0.430 \pm 0.039$ & $22 / 13$ \\
\hline & 2.95 & 0.43 & $0.950 \pm 0.004$ & $0.113 \pm 0.002$ & 5 & $2.000 \pm 0.090$ & $0.438 \pm 0.008$ & $10 / 10$ \\
\hline & 2.95 & 0.49 & $0.950 \pm 0.012$ & $0.124 \pm 0.008$ & 5 & $1.900 \pm 0.070$ & $0.480 \pm 0.031$ & $15 / 12$ \\
\hline & 3.35 & 0.43 & $0.950 \pm 0.010$ & $0.124 \pm 0.005$ & 5 & $0.300 \pm 0.020$ & $0.480 \pm 0.019$ & $11 / 10$ \\
\hline & 3.35 & 0.49 & $0.950 \pm 0.010$ & $0.124 \pm 0.003$ & 5 & $1.100 \pm 0.080$ & $0.480 \pm 0.012$ & $9 / 10$ \\
\hline & 3.85 & 0.43 & $0.950 \pm 0.006$ & $0.128 \pm 0.006$ & 5 & $0.400 \pm 0.030$ & $0.496 \pm 0.023$ & $10 / 12$ \\
\hline & 3.85 & 0.49 & $0.950 \pm 0.003$ & $0.129 \pm 0.002$ & 5 & $0.750 \pm 0.040$ & $0.500 \pm 0.008$ & $8 / 9$ \\
\hline & 3.85 & 0.55 & $0.950 \pm 0.003$ & $0.131 \pm 0.002$ & 5 & $1.500 \pm 0.080$ & $0.507 \pm 0.008$ & $4 / 11$ \\
\hline & 4.35 & 0.55 & $0.950 \pm 0.002$ & $0.135 \pm 0.002$ & 5 & $1.200 \pm 0.060$ & $0.523 \pm 0.008$ & $1 /(4)$ \\
\hline
\end{tabular}

$$
\begin{aligned}
|t|= & \left|\left(E_{1}-E_{3}\right)^{2}-\left(\vec{p}_{1}-\vec{p}_{3}\right)^{2}\right| \\
= & \mid m_{1}^{2}+m_{3}^{2}-2 E_{1} \sqrt{\left(\frac{p_{3 T}}{\sin \theta}\right)^{2}+m_{3}^{2}} \\
& +2 \sqrt{E_{1}^{2}-m_{1}^{2}} \frac{p_{3 T}}{\tan \theta} \mid,
\end{aligned}
$$

where $E_{1}$ and $E_{3}, \vec{p}_{1}$ and $\vec{p}_{3}$, as well as $m_{1}$ and $m_{3}$ are the energy, momentum, and rest mass of particles 1 and 3 , respectively. In addition, $p_{3 T}$ referred to be perpendicular to the $\mathrm{O} z$ direction component of the transverse momentum of particle 3 , which obeys the Erlang distribution, that is Eq. 2 in which $p_{T}=p_{3 T}$.

In this paper, the squared momentum transfer spectra of light meson at different squared photon virtuality $Q^{2}$ and Bjorken variable $x_{B}$ are fitted by calculated results with the Monte Carlo method. Here, $Q^{2}$ is a reflection of hard scale of reaction [47-54]. The harder the reaction is, the higher the excitation degree is. In fact, $Q^{2}$ is the absolute value of the squared mass of $\gamma^{*}$ (particle 2) that is exchanged between the scattered electron and the target proton (particle 1), and it effectively represents the transverse size of the probe [38]. In addition, $-Q^{2}$ is also the squared momentum transfer to the target proton (particle 1) by the scattered electron [37].

As for the Bjorken variable $x_{B}$, it represents contrarily the momentum of particle 1 . The lower the $x_{B}$ is, the higher the momentum of particle 1 is. Generally, $x_{B}=Q^{2} /\left(2 P_{2}\right.$. $\left.\sqrt{-Q^{2}}\right) \propto Q$ [37]. In a symmetric frame, importing $\xi^{\prime}$ as skewness, it is half of the longitudinal momentum fraction transferred to the struck parton. The skewness $\xi^{\prime}$ can be used to express $x_{B}$ approximately. That is $x_{B} \approx 2 \xi^{\prime} /\left(1+\xi^{\prime}\right)$ [37]. 


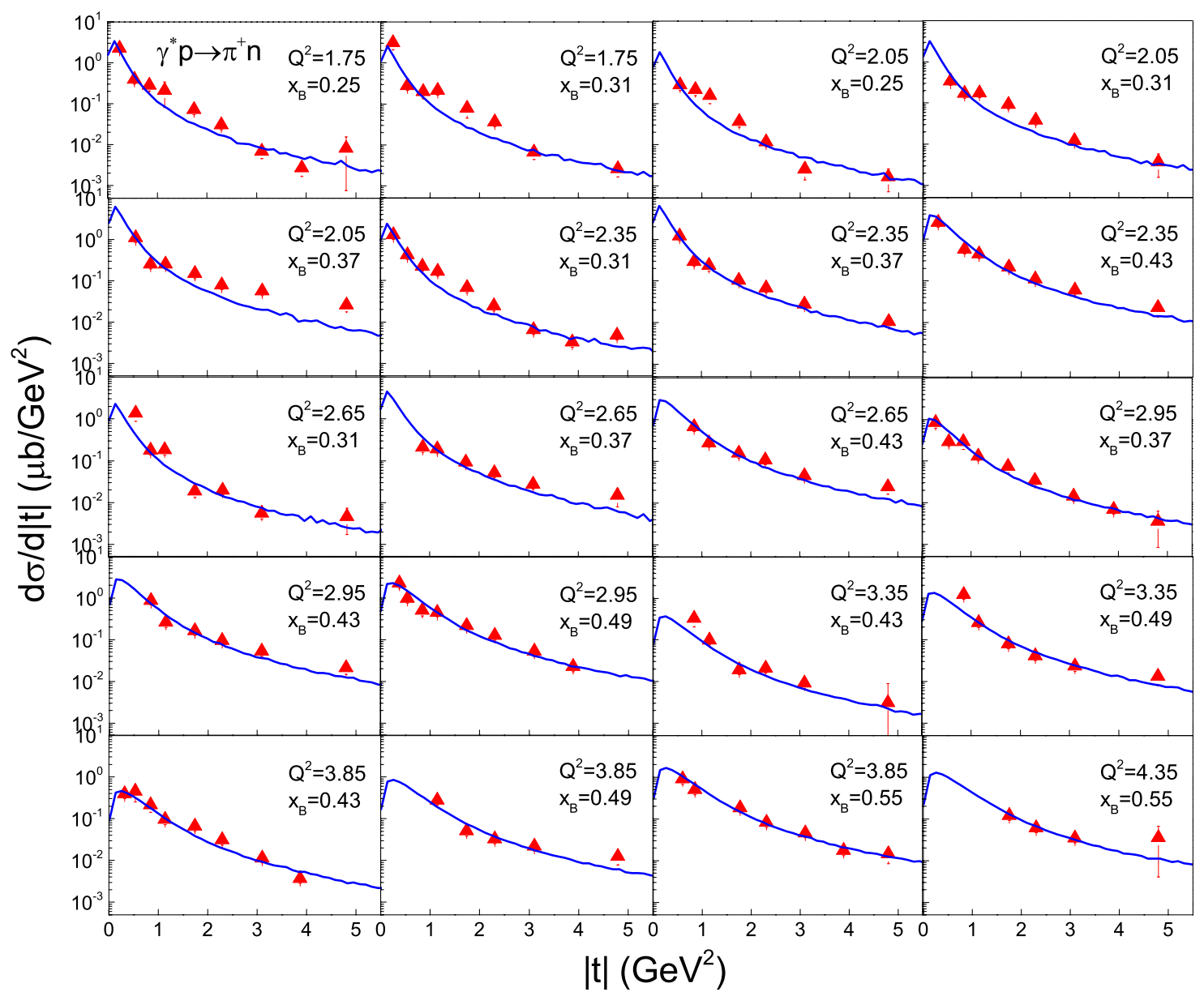

FIGURE 2 | The differential cross-section $d \sigma / d|t|$ in $|t|$ of $\gamma^{*} p \rightarrow \pi^{+} n$ process produced in ep collisions at beam energy of 6 GeV at different $Q^{2}$ and $x_{B}$ shown in the panels. The symbols represent the experimental data measured by the CLAS Collaboration [38] and the curves are the statistical results obtained as those in Figure $\mathbf{1}$

\subsection{The Process of Monte Carlo Calculations}

In the calculations of squared momentum transfer, the analytical expression of $p_{T}$ distribution is difficult to be transformed to that of squared momentum transfer distribution directly by using Eq. 9. Alternatively, we may use the Monte Carlo method to transform $p_{T}$ to squared momentum transfer. Let $R_{1,2}$ and $r_{1,2,3, \ldots, n_{s}}$ be random numbers distributed evenly in $[0,1]$. Then, many concrete transverse momentum $p_{3 T}$ satisfied with Eq. 2 and $\theta$ are produced. Other quantities such as $E_{1}, m_{1}$, and $m_{3}$ in the equation are fixed, though $E_{1}$ is treated as a parameter in the present work.

Generally, we may solve the equation

$$
\int_{0}^{p_{T}} f\left(p_{T}^{\prime}\right) d p_{T}^{\prime}<R_{1}<\int_{0}^{p_{T}+\delta p_{T}} f\left(p_{T}^{\prime}\right) d p_{T}^{\prime},
$$

where $\delta p_{T}$ is a small shift relative to $p_{T}$. Conveniently, there is a simpler expression due to Eqs 1, 2. In fact, solving the equation

$$
\int_{0}^{p_{t j}} f\left(p_{t j}^{\prime}\right) d p_{t j}^{\prime}=r_{j} \quad\left(j=1,2,3, \ldots, n_{s}\right),
$$

we have

$$
p_{t j}=-\left\langle p_{t}\right\rangle \ln r_{j} \quad\left(j=1,2,3, \ldots, n_{s}\right) .
$$

The simpler expression is

$$
p_{T}=\sum_{j=1}^{n_{s}} p_{t j}=-\left\langle p_{t}\right\rangle \sum_{j=1}^{n_{s}} \ln r_{j}=-\left\langle p_{t}\right\rangle \ln \left(\prod_{j=1}^{n_{s}} r_{j}\right) .
$$

The distribution of $\theta$ satisfies with the half-sine function

$$
f_{\theta}(\theta)=\frac{1}{2} \sin \theta
$$

which is obtained under the assumption of isotropic emission in the source's rest frame. Solving the equation

$$
\int_{0}^{\theta} f_{\theta}\left(\theta^{\prime}\right) d \theta^{\prime}=R_{2}
$$




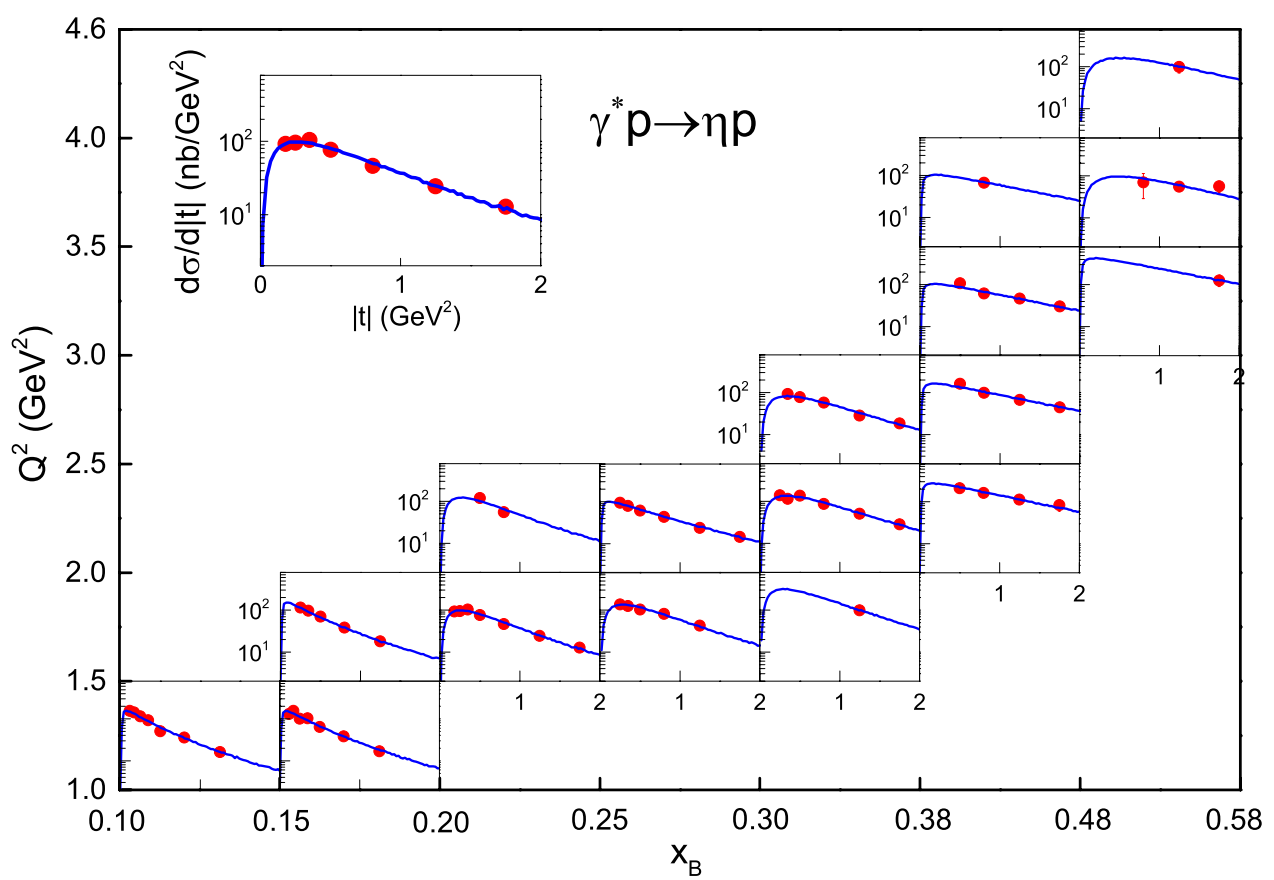

FIGURE 3 | The differential cross-section $d \sigma / d|t|$ in $|t|$ of $\gamma^{*} p \rightarrow \eta p$ process produced in ep collisions at beam energy of $5.75 \mathrm{GeV}$ in different $Q^{2}$ and $x_{B}$ ranges shown in the panels. As an example, the sample at the top-left sub-panel shows repeatedly the result in $1.5<Q^{2}<2.0 \mathrm{GeV}^{2}$ and $0.20<x_{B}<0.25$. The symbols represent the experimental data measured by the CLAS Collaboration [39] and the curves are the statistical results obtained as those in Figure 1.

we have

$$
\theta=2 \arcsin \left(\sqrt{R_{2}}\right)
$$

which is needed in the calculations.

We have check the consistency and correctness of the above expressions in the Monte Carlo method in terms of illustration which is not presented here. After obtaining concrete values of $p_{3 T}$ and $\theta$, and using $E_{1}, m_{1}$, and $m_{3}$, the value of $|t|$ can be obtained from Eq. 9. Through repeating the calculations many times, the distribution of $|t|$ is obtained statistically. Based on the method of least squares, the parameter $\left\langle p_{t}\right\rangle$ and $n_{s}$ are extracted naturally. Meanwhile, $T_{i}$ can be obtained from Eq. 4 and $\left\langle p_{T}\right\rangle \quad\left(\left\langle p_{T}^{2}\right\rangle\right)$ can be obtained from Eq. 3 [(5)] or from the statistics. The errors of parameters are obtained by the general method of statistical analysis.

It should be noted that the above Monte Carlo calculation is only performed in the transformation from transverse momentum to $|t|$, in which the physics process such as the radiative corrections for reactions induced by electrons has been taken into account naturally. In fact, the effects of the mentioned process and all other processes are included in the Erlang distribution which is a result of multifactor interactions. In other words, the Monte Carlo calculation used here is not a simulation for the system evolution from initial to final stages, but the numerical transformation in the final stage.

\section{RESULTS AND DISCUSSION}

\subsection{Comparison With Data}

Figure 1 shows the differential cross-section, $d \sigma / d|t|$, in squared momentum transfer $|t|$ of $\gamma^{*} p \rightarrow \pi^{0} p$ process produced in
$5.75 \mathrm{GeV}$ electron beam induced collisions in a $2.5 \mathrm{~cm}$ long liquid-hydrogen target ( $e p$ collisions at beam energy of $5.75 \mathrm{GeV}$ ) in different ranges of squared photon virtuality, 1.0 $<Q^{2}<1.5,1.5<Q^{2}<2.0,2.0<Q^{2}<2.5,2.5<Q^{2}<3.0,3.0<Q^{2}<$ $3.5,3.5<Q^{2}<4.0$, and $4.0<Q^{2}<4.6 \mathrm{GeV}^{2}$, from bottom to up sub-panels, as well as in different ranges of Bjorken variable, $0.10<x_{B}<0.15,0.15<x_{B}<0.20,0.20<x_{B}<0.25,0.25<x_{B}<$ $0.30,0.30<x_{B}<0.38,0.38<x_{B}<0.48$, and $0.48<x_{B}<0.58$, from left to right sub-panels. The sample at the top-left subpanel shows repeatedly the result in the range of squared photon virtuality, $1.5<Q^{2}<2.0 \mathrm{GeV}^{2}$, and the range of Bjorken variable, $0.20<x_{B}<0.25$, as an example. The symbols represent the experimental data measured by the CLAS Collaboration [37] and the curves are the statistical results of squared momentum transfer $|t|$.

In Eq. 9, $p_{3 T}$ satisfies the Erlang distribution (Eq. 2) and we obtain it by the Monte Carlo method (Eq. 13). Then, the squared momentum transfer $|t|$ is obtained statistically. In the fitting process, two main parameters, i.e., the average transverse momentum $\left\langle p_{t}\right\rangle$ contributed by each participant parton and the number $n_{s}$ of participant partons are extracted naturally. To obtain a better fit result, $E_{1}$ is extracted as an insensitive parameter. In addition, a non-free parameter is the normalization constant $\sigma_{0}$. The values of parameters with selection condition $\left(Q^{2}\right.$ and $\left.x_{B}\right), \chi^{2}$, and the number of degree of freedom (ndof) are listed in Table 1, where the number of parameters is always 4 which includes $E_{1},\left\langle p_{t}\right\rangle, n_{s}$, and $\sigma_{0}$. In the case of ndof being less than or equal to the number of parameters, we obtain the curve from a "prediction" 
TABLE 2 | Same as Table 1, but corresponding to the curves in Figure 3, 4

\begin{tabular}{|c|c|c|c|c|c|c|c|c|}
\hline Collisions & $\begin{array}{c}Q^{2} \\
(\mathrm{GeV})\end{array}$ & $x_{B}$ & $\begin{array}{c}E_{1} \\
(\mathrm{GeV})\end{array}$ & $\begin{array}{c}\left\langle p_{t}\right\rangle \\
(\mathrm{GeV} / c)\end{array}$ & $n_{s}$ & $\begin{array}{c}\sigma_{0} \\
(\mu b)\end{array}$ & $\begin{array}{c}T_{i} \\
(\mathrm{GeV})\end{array}$ & $\begin{array}{c}\chi^{2} / \text { ndof } \\
\text { (nop) }\end{array}$ \\
\hline \multirow[t]{17}{*}{$\gamma^{*} p \rightarrow \eta p$} & $(1.0,1.5)$ & $(0.10,0.15)$ & $0.955 \pm 0.005$ & $0.188 \pm 0.001$ & 3 & $0.093 \pm 0.004$ & $0.449 \pm 0.003$ & $7 / 11$ \\
\hline & $(1.0,1.5)$ & $(0.15,0.20)$ & $0.955 \pm 0.003$ & $0.195 \pm 0.003$ & 3 & $0.098 \pm 0.005$ & $0.466 \pm 0.008$ & $5 / 11$ \\
\hline & $(1.5,2.0)$ & $(0.15,0.20)$ & $0.955 \pm 0.002$ & $0.196 \pm 0.001$ & 3 & $0.098 \pm 0.007$ & $0.468 \pm 0.003$ & $1 / 9$ \\
\hline & $(1.5,2.0)$ & $(0.20,0.25)$ & $0.955 \pm 0.001$ & $0.140 \pm 0.001$ & 5 & $0.098 \pm 0.003$ & $0.557 \pm 0.004$ & $3 / 11$ \\
\hline & $(2.0,2.5)$ & $(0.20,0.25)$ & $0.955 \pm 0.010$ & $0.142 \pm 0.002$ & 5 & $0.125 \pm 0.008$ & $0.566 \pm 0.008$ & $2 /(2)$ \\
\hline & $(1.5,2.0)$ & $(0.25,0.30)$ & $0.955 \pm 0.002$ & $0.148 \pm 0.001$ & 5 & $0.147 \pm 0.008$ & $0.589 \pm 0.004$ & $3 / 9$ \\
\hline & $(2.0,2.5)$ & $(0.25,0.30)$ & $0.955 \pm 0.005$ & $0.248 \pm 0.001$ & 3 & $0.100 \pm 0.006$ & $0.592 \pm 0.003$ & $1 / 11$ \\
\hline & $(1.5,2.0)$ & $(0.30,0.38)$ & $0.955 \pm 0.010$ & $0.150 \pm 0.010$ & 5 & $0.354 \pm 0.015$ & $0.597 \pm 0.039$ & $0.04 /(1)$ \\
\hline & $(2.0,2.5)$ & $(0.30,0.38)$ & $0.955 \pm 0.004$ & $0.160 \pm 0.001$ & 5 & $0.170 \pm 0.005$ & $0.637 \pm 0.004$ & $4 / 10$ \\
\hline & $(2.5,3.0)$ & $(0.30,0.38)$ & $0.955 \pm 0.003$ & $0.162 \pm 0.002$ & 5 & $0.105 \pm 0.006$ & $0.644 \pm 0.008$ & $3 / 9$ \\
\hline & $(2.0,2.5)$ & $(0.38,0.48)$ & $0.955 \pm 0.002$ & $0.300 \pm 0.001$ & 3 & $0.390 \pm 0.018$ & $0.716 \pm 0.003$ & $0.3 /(1)$ \\
\hline & $(2.5,3.0)$ & $(0.38,0.48)$ & $0.955 \pm 0.002$ & $0.305 \pm 0.002$ & 3 & $0.240 \pm 0.013$ & $0.728 \pm 0.005$ & $2 /(4)$ \\
\hline & $(3.0,3.5)$ & $(0.38,0.48)$ & $0.955 \pm 0.002$ & $0.308 \pm 0.002$ & 3 & $0.155 \pm 0.012$ & $0.735 \pm 0.005$ & $2 /(4)$ \\
\hline & $(3.5,4.0)$ & $(0.38,0.48)$ & $0.955 \pm 0.002$ & $0.314 \pm 0.002$ & 3 & $0.160 \pm 0.007$ & $0.750 \pm 0.005$ & $0.01 /(4)$ \\
\hline & $(3.0,3.5)$ & $(0.48,0.58)$ & $0.955 \pm 0.003$ & $0.315 \pm 0.003$ & 3 & $0.660 \pm 0.030$ & $0.752 \pm 0.008$ & $0.01 /(1)$ \\
\hline & $(3.5,4.0)$ & $(0.48,0.58)$ & $0.955 \pm 0.005$ & $0.193 \pm 0.004$ & 5 & $0.165 \pm 0.005$ & $0.768 \pm 0.016$ & $2 /(3)$ \\
\hline & $(4.0,4.6)$ & $(0.48,0.58)$ & $0.955 \pm 0.004$ & $0.195 \pm 0.005$ & 5 & $0.280 \pm 0.010$ & $0.776 \pm 0.020$ & $0.01 /(1)$ \\
\hline \multirow{27}{*}{$\gamma^{\star} p \rightarrow \rho^{0} p$} & $(1.6,1.9)$ & $(0.16,0.22)$ & $0.960 \pm 0.005$ & $0.112 \pm 0.001$ & 3 & $1.200 \pm 0.060$ & $0.274 \pm 0.003$ & $4 / 11$ \\
\hline & $(1.6,1.9)$ & $(0.22,0.28)$ & $0.960 \pm 0.004$ & $0.115 \pm 0.001$ & 3 & $2.300 \pm 0.090$ & $0.282 \pm 0.002$ & $2 / 10$ \\
\hline & $(1.9,2.2)$ & $(0.22,0.28)$ & $0.960 \pm 0.003$ & $0.119 \pm 0.002$ & 3 & $1.200 \pm 0.050$ & $0.291 \pm 0.005$ & $1 / 11$ \\
\hline & $(2.2,2.5)$ & $(0.22,0.28)$ & $0.960 \pm 0.003$ & $0.120 \pm 0.001$ & 3 & $1.000 \pm 0.030$ & $0.294 \pm 0.003$ & $2 / 11$ \\
\hline & $(1.6,1.9)$ & $(0.28,0.34)$ & $0.960 \pm 0.005$ & $0.119 \pm 0.004$ & 3 & $6.100 \pm 0.210$ & $0.291 \pm 0.010$ & $8 / 10$ \\
\hline & $(1.9,2.2)$ & $(0.28,0.34)$ & $0.960 \pm 0.007$ & $0.121 \pm 0.004$ & 3 & $2.200 \pm 0.080$ & $0.296 \pm 0.010$ & $5 / 11$ \\
\hline & $(2.2,2.5)$ & $(0.28,0.34)$ & $0.960 \pm 0.003$ & $0.126 \pm 0.003$ & 3 & $1.400 \pm 0.040$ & $0.309 \pm 0.008$ & $4 / 11$ \\
\hline & $(2.5,2.8)$ & $(0.28,0.34)$ & $0.960 \pm 0.010$ & $0.127 \pm 0.004$ & 3 & $1.000 \pm 0.030$ & $0.311 \pm 0.010$ & $2 / 11$ \\
\hline & $(1.9,2.2)$ & $(0.34,0.40)$ & $0.960 \pm 0.004$ & $0.163 \pm 0.005$ & 3 & $4.000 \pm 0.170$ & $0.399 \pm 0.012$ & $7 / 11$ \\
\hline & $(2.2,2.5)$ & $(0.34,0.40)$ & $0.960 \pm 0.020$ & $0.168 \pm 0.014$ & 3 & $1.700 \pm 0.090$ & $0.412 \pm 0.034$ & $3 / 11$ \\
\hline & $(2.5,2.8)$ & $(0.34,0.40)$ & $0.960 \pm 0.016$ & $0.175 \pm 0.005$ & 3 & $1.250 \pm 0.070$ & $0.429 \pm 0.013$ & $2 / 11$ \\
\hline & $(2.8,3.1)$ & $(0.34,0.40)$ & $0.960 \pm 0.006$ & $0.177 \pm 0.003$ & 3 & $1.000 \pm 0.080$ & $0.434 \pm 0.008$ & $2 / 11$ \\
\hline & $(3.1,3.6)$ & $(0.34,0.40)$ & $0.960 \pm 0.004$ & $0.178 \pm 0.002$ & 3 & $0.700 \pm 0.030$ & $0.436 \pm 0.005$ & $2 / 11$ \\
\hline & $(2.2,2.5)$ & $(0.40,0.46)$ & $0.960 \pm 0.020$ & $0.185 \pm 0.010$ & 3 & $3.300 \pm 0.160$ & $0.453 \pm 0.024$ & $9 / 11$ \\
\hline & $(2.5,2.8)$ & $(0.40,0.46)$ & $0.960 \pm 0.008$ & $0.190 \pm 0.006$ & 3 & $2.100 \pm 0.140$ & $0.465 \pm 0.014$ & $4 / 11$ \\
\hline & $(2.8,3.1)$ & $(0.40,0.46)$ & $0.960 \pm 0.020$ & $0.215 \pm 0.008$ & 3 & $1.300 \pm 0.090$ & $0.527 \pm 0.020$ & $0.4 / 11$ \\
\hline & $(3.1,3.6)$ & $(0.40,0.46)$ & $0.960 \pm 0.010$ & $0.217 \pm 0.006$ & 3 & $0.800 \pm 0.060$ & $0.532 \pm 0.015$ & $0.7 / 11$ \\
\hline & $(3.6,4.1)$ & $(0.40,0.46)$ & $0.960 \pm 0.015$ & $0.218 \pm 0.020$ & 3 & $0.700 \pm 0.040$ & $0.534 \pm 0.049$ & $2 / 11$ \\
\hline & $(2.8,3.1)$ & $(0.46,0.52)$ & $0.960 \pm 0.020$ & $0.250 \pm 0.010$ & 3 & $2.000 \pm 0.150$ & $0.612 \pm 0.025$ & $8 / 11$ \\
\hline & $(3.1,3.6)$ & $(0.46,0.52)$ & $0.960 \pm 0.002$ & $0.254 \pm 0.002$ & 3 & $0.900 \pm 0.060$ & $0.622 \pm 0.005$ & $1 / 11$ \\
\hline & $(3.6,4.1)$ & $(0.46,0.52)$ & $0.960 \pm 0.010$ & $0.270 \pm 0.006$ & 3 & $0.800 \pm 0.050$ & $0.661 \pm 0.015$ & $1 / 11$ \\
\hline & $(4.1,4.6)$ & $(0.46,0.52)$ & $0.960 \pm 0.009$ & $0.272 \pm 0.002$ & 3 & $0.800 \pm 0.030$ & $0.666 \pm 0.005$ & $2 / 11$ \\
\hline & $(3.6,4.1)$ & $(0.52,0.58)$ & $0.960 \pm 0.020$ & $0.300 \pm 0.020$ & 3 & $1.200 \pm 0.070$ & $0.735 \pm 0.049$ & $6 / 11$ \\
\hline & $(4.1,4.6)$ & $(0.52,0.58)$ & $0.960 \pm 0.020$ & $0.400 \pm 0.030$ & 3 & $1.000 \pm 0.050$ & $0.980 \pm 0.073$ & $1 / 10$ \\
\hline & $(4.1,4.6)$ & $(0.58,0.64)$ & $0.960 \pm 0.020$ & $0.410 \pm 0.030$ & 3 & $1.000 \pm 0.040$ & $1.004 \pm 0.074$ & $6 / 10$ \\
\hline & $(4.6,5.1)$ & $(0.58,0.64)$ & $0.960 \pm 0.010$ & $0.420 \pm 0.020$ & 3 & $0.900 \pm 0.030$ & $1.029 \pm 0.049$ & $6 / 10$ \\
\hline & $(5.1,5.6)$ & $(0.64,0.70)$ & $0.960 \pm 0.010$ & $0.430 \pm 0.010$ & 3 & $0.900 \pm 0.040$ & $1.053 \pm 0.025$ & $0.4 /(4)$ \\
\hline
\end{tabular}

or extrapolation based on other reasonable fits in which the tendency of parameters is available. Meanwhile, in these cases, the number of points (nop) is given in a bracket to replace ndof in the table. One can see that the values of $\chi^{2}$ are small in most cases, though the (necessary) dense log scale is not easy to judge. The model results are in agreement with the experimental data. From the values of parameters, the average transverse momentum $\left\langle p_{T}\right\rangle$ and initial temperature $T_{i}$ are obtained naturally.

Figure 2 presents the differential cross-section, $d \sigma / d|t|$, in $|t|$ of $\gamma^{*} p \rightarrow \pi^{+} n$ process produced in ep collisions at beam energy of $6 \mathrm{GeV}$ at different squared photon virtuality, $Q^{2}=1.75,2.05,2.35$, $2.65,2.95,3.35,3.85$, and $4.35 \mathrm{GeV}^{2}$, as well as at different Bjorken variable, $x_{B}=0.25,0.31,0.37,0.43,0.49$, and 0.55 .
The symbols represent the experimental data measured by the CLAS Collaboration [38]. As those in Figure 1, the curves in Figure 2 are also the statistical results of $|t|$ in which $p_{3 T}$ satisfies the Erlang distribution and is obtained by the Monte Carlo method. The values of parameters with selection condition $\left(Q^{2}\right.$ and $\left.x_{B}\right), \chi^{2}$, and ndof are listed in Table 1 . One can see that the model results are in agreement with the experimental data.

Figure 3 displays the differential cross-section, $d \sigma / d|t|$, in $|t|$ of $\gamma^{*} p \rightarrow \eta p$ process produced in ep collisions at beam energy of $5.75 \mathrm{GeV}$ in different $Q^{2}$ and $x_{B}$ ranges shown in the panel. As an example, the sample at the top-left sub-panel shows repeatedly the result in $1.5<Q^{2}<2.0 \mathrm{GeV}^{2}$ and $0.20<x_{B}$ $<0.25$. The symbols represent the experimental data measured 


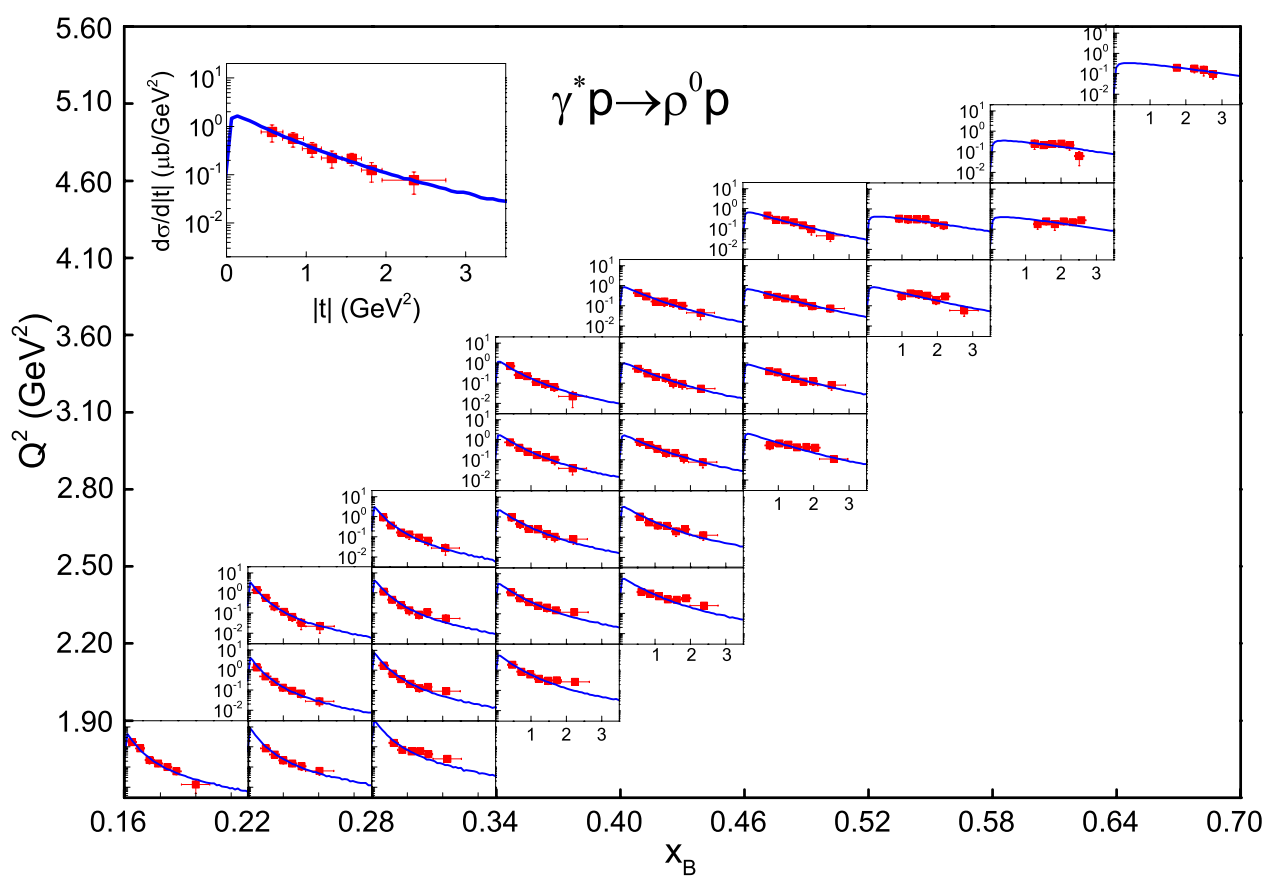

FIGURE $4 \mid$ The differential cross-section $d \sigma / d|t|$ in $|t|$ of $\gamma^{*} p \rightarrow \rho^{0} p$ process produced in ep collisions at beam energy of 5.754 GeV in different $Q^{2}$ and $x_{B}$ ranges shown in the panels. As an example, the sample at the top-left sub-panel shows repeatedly the result in $2.8<Q^{2}<3.1 \mathrm{GeV}^{2}$ and $0.40<x_{B}<0.46$. The symbols represent the experimental data measured by the CLAS Collaboration [40] and the curves are the statistical results obtained as those in Figure 1.

by the CLAS Collaboration [39]. The curves are the statistical results of $|t|$ in which $p_{3 T}$ satisfies the Erlang distribution and is obtained by the Monte Carlo method. The values of parameters with selection condition $\left(Q^{2}\right.$ and $\left.x_{B}\right), \chi^{2}$, and ndof are listed in Table 2. One can see that the model results are in agreement with the experimental data.

Similar to Figures 1-3, Figure 4 presents the differential crosssection, $d \sigma / d|t|$, in $|t|$ of $\gamma^{*} p \rightarrow \rho^{0} p$ process produced in $e p$ collisions at beam energy of $5.754 \mathrm{GeV}$ in different $Q^{2}$ and $x_{B}$ ranges shown in the panel. As an example, the sample at the top-left sub-panel shows repeatedly the result in $2.8<Q^{2}<3.1 \mathrm{GeV}^{2}$ and $0.40<x_{B}<0.46$ range. The symbols represent the experimental data measured by the CLAS Collaboration [40]. The curves are the statistical results of $|t|$ in which $p_{3 T}$ satisfies the Erlang distribution and is obtained by the Monte Carlo method. The values of parameters with selection condition $\left(Q^{2}\right.$ and $\left.x_{B}\right)$, $\chi^{2}$, and ndof are listed in Table 2. One can see that the model results are in agreement with the experimental data.

\subsection{Parameter Tendency and Discussion}

In Figures 1-4, the cross-sections for $\pi^{0} p, \pi^{+} n, \eta p$, and $\rho^{0} p$ are fitted to show some differences in concrete values and parameters, and some common features among them in the tendency of curves also appear. This is caused by the fact that different channels have different fraction ratios, and all of them are from the same ep collisions, though the collision energies are slightly different.

The dependences of $\left\langle p_{T}\right\rangle(\mathrm{A}, \mathrm{C}, \mathrm{E}, \mathrm{G})$ and $T_{i}(\mathrm{~B}, \mathrm{D}, \mathrm{F}, \mathrm{H})$ on $Q^{2}$ in $\gamma^{*} p$ collisions with different emitted channels $\left[\pi^{0} p(\mathrm{~A}, \mathrm{~B})\right.$, $\pi^{+} n(\mathrm{C}, \mathrm{D}), \eta p(\mathrm{E}, \mathrm{F})$, and $\left.\rho^{0} p(\mathrm{G}, \mathrm{H})\right]$ are shown in Figure 5, where $\left\langle p_{T}\right\rangle=n_{s}\left\langle p_{t}\right\rangle$ due to Tables 1, 2 and the values of $T_{i}$ are from Tables 1, 2. Different symbols represent the results for different $x_{B}$. One can see that $\left\langle p_{T}\right\rangle$ and $T_{i}$ increase generally with an increase in $Q^{2}$. Because $Q^{2}$ represents the hard scale (violent degree) of collisions and a harder scale results in a higher excitation degree, it is natural that larger $\left\langle p_{T}\right\rangle$ and $T_{i}$ appear at higher $Q^{2}$.

Figure 6 is similar to Figure 5, but it shows the dependences of $\left\langle p_{T}\right\rangle(\mathrm{A}, \mathrm{C}, \mathrm{E}, \mathrm{G})$ and $T_{i}(\mathrm{~B}, \mathrm{D}, \mathrm{F}, \mathrm{H})$ on $x_{B}$ in $\gamma^{*} p$ collisions with emitted channels $\pi^{0} p(\mathrm{~A}, \mathrm{~B}), \pi^{+} n(\mathrm{C}, \mathrm{D}), \eta p(\mathrm{E}, \mathrm{F})$, and $\rho^{0} p(\mathrm{G}, \mathrm{H})$. Different symbols represent the results for different $Q^{2}$. One can see that $\left\langle p_{T}\right\rangle$ and $T_{i}$ increase generally with an increase in $x_{B}$. Because $x_{B} \propto Q$, we may think that $x_{B}$ also represents the hard scale of collisions and a harder scale results in a higher excitation degree. It is understandable that larger $\left\langle p_{T}\right\rangle$ and $T_{i}$ appear at higher $x_{B}$.

In addition, $x_{B}$ also represents the longitudinal momentum fraction transferred to the struck parton. In the considered $\gamma^{\star} p \rightarrow$ meson + nucleon process in ep collisions at given energy, the larger $x_{B}$ means the larger longitudinal momentum transfer to the struck parton or the system, and hence the more energy deposited to the system. The system naturally stays at higher excitation degree. As a result, larger $\left\langle p_{T}\right\rangle$ and $T_{i}$ are observed.

Generally, $\left\langle p_{T}\right\rangle>T_{i} \geq T_{c h} \geq T_{0}$. If the evolution time of the system is 0 , that is if the initial-state, chemical freeze-out, and kinetic freeze-out happen simultaneously, we have $T_{i}=T_{c h}=T_{0}$. If the evolution time is not negligible, we have $T_{i}>T_{c h}>T_{0}$. The difference between $\left\langle p_{T}\right\rangle$ and temperature is explained as the contribution of flow effect. According to Ref. [55], in the finalstate, the expected real $T_{0} \approx\left\langle p_{T}\right\rangle / 3.07$. Then, we have the contribution of flow effect to be $\left\langle p_{T}\right\rangle-T_{0} \approx 2.07\left\langle p_{T}\right\rangle / 3.07$. 

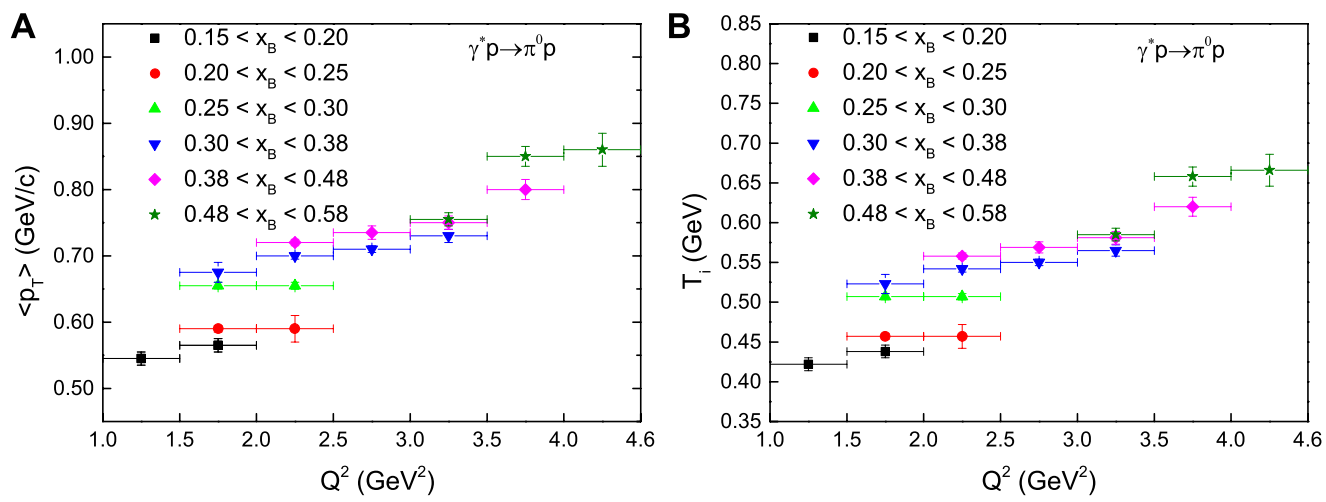

C

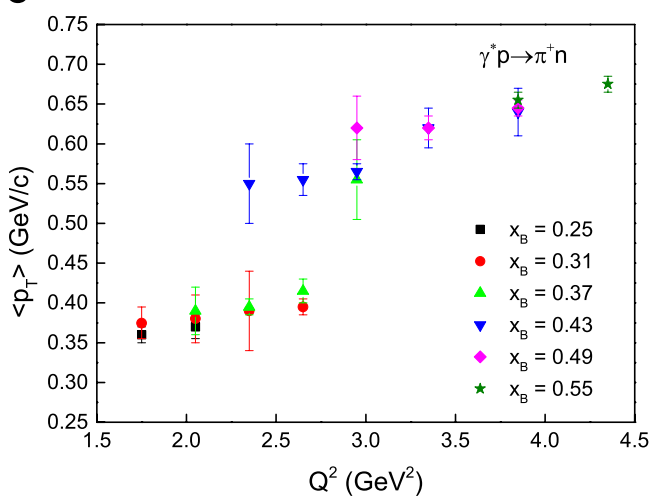

E

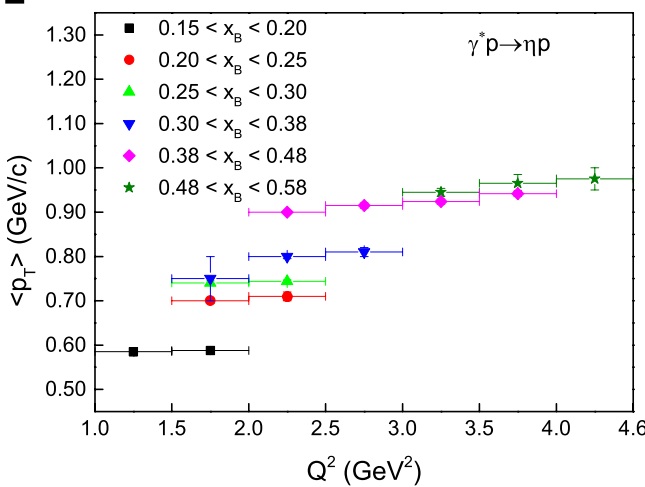

G

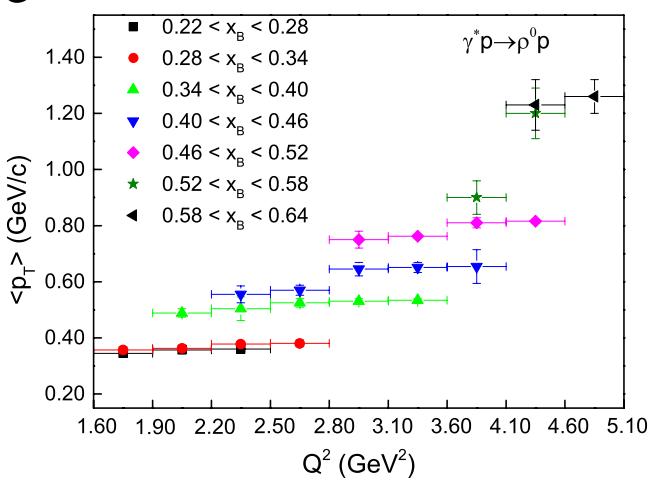

D

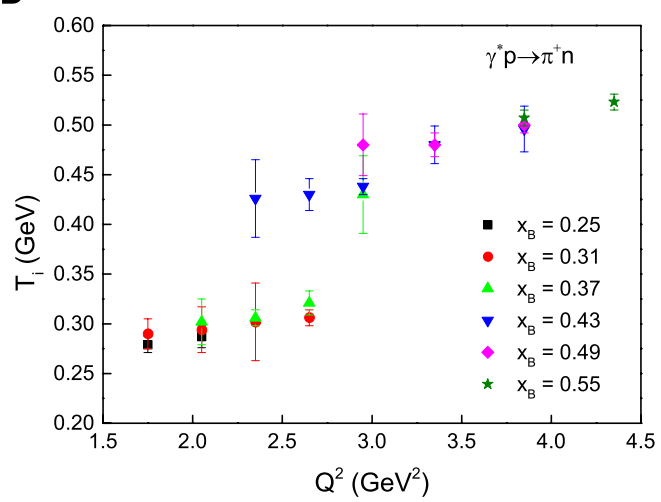

$\mathbf{F}$

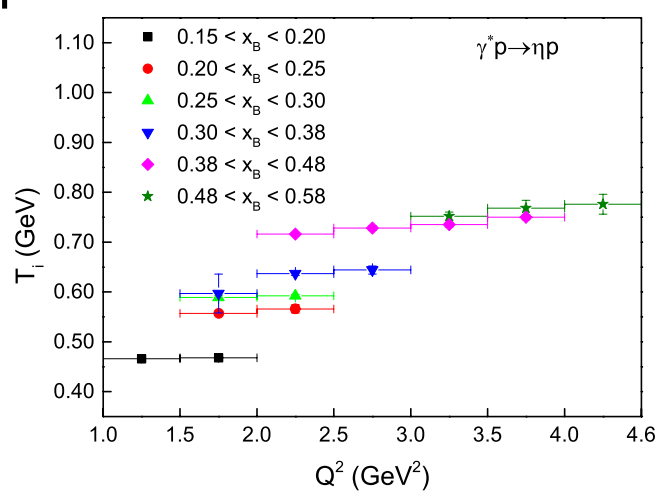

H

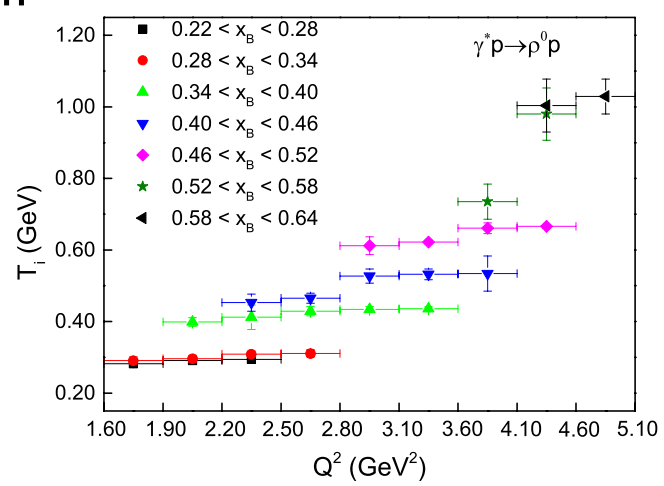

FIGURE $5 \mid$ The dependences of $\left\langle p_{T}\right\rangle(\mathbf{A}, \mathbf{C}, \mathbf{E}, \mathbf{G})$ and $T_{i}(\mathbf{B}, \mathbf{D}, \mathbf{F}, \mathbf{H})$ on $Q^{2}$ in $\gamma^{\star} p$ collisions with emitted channels $\pi^{0} p(\mathbf{A}, \mathbf{B}), \pi^{+} n(\mathbf{C}, \mathbf{D}), \eta p(\mathbf{E}, \mathbf{F})$, and $\rho^{0} p(\mathbf{G}, \mathbf{H})$. Different symbols represent the results for different $x_{B}$. 


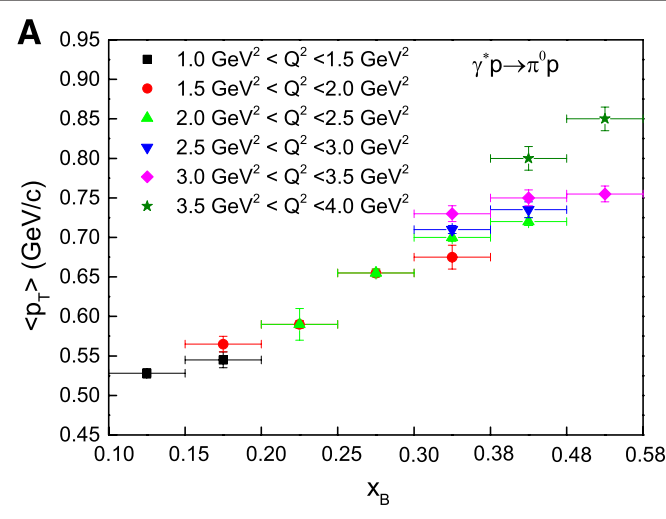

C

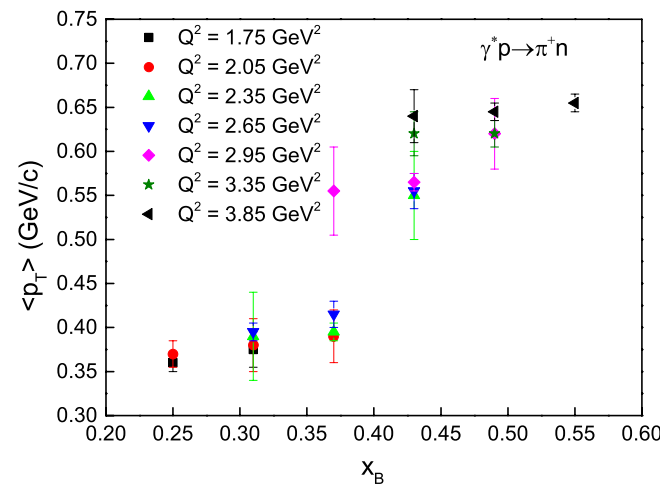

E

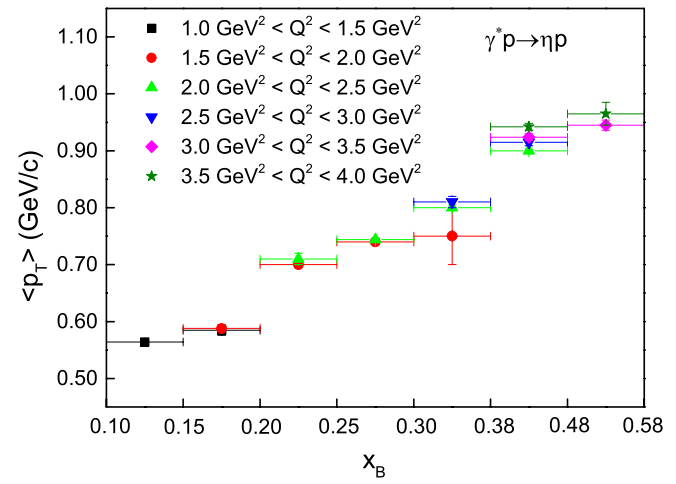

G

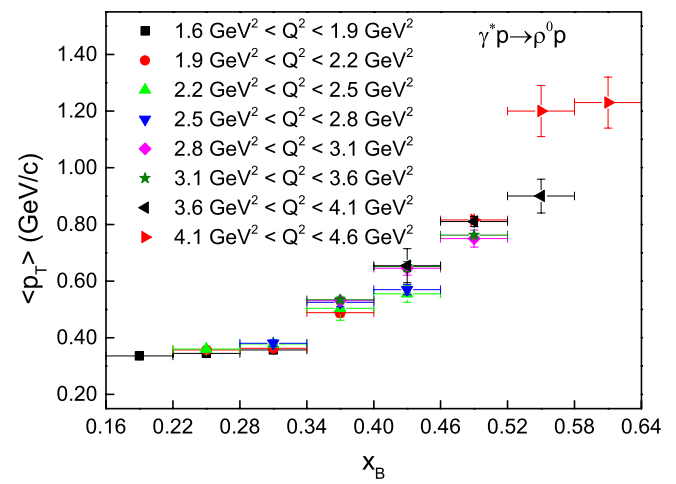

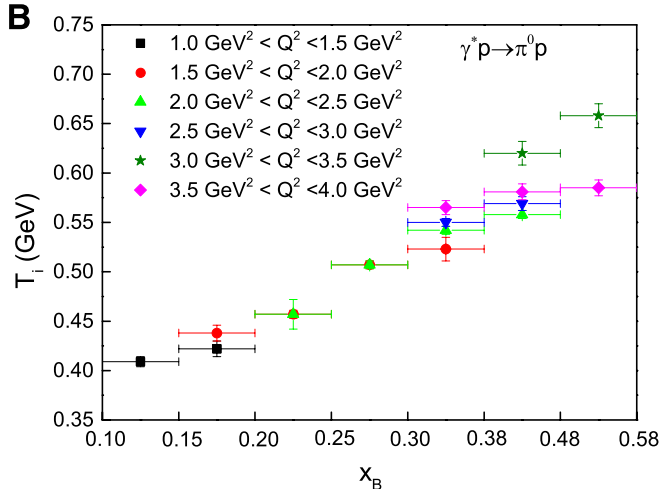

D

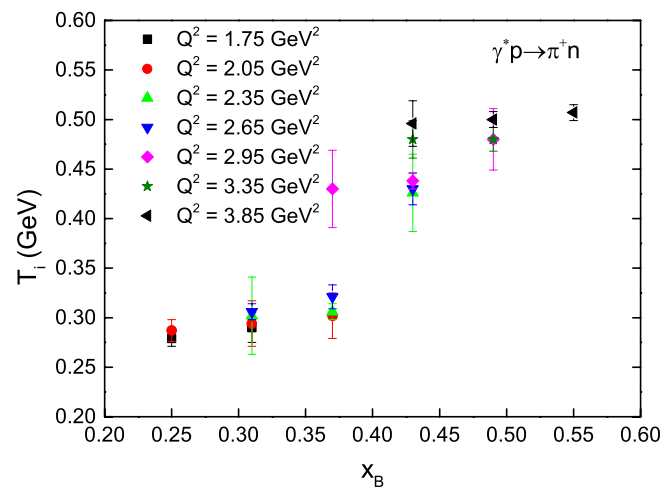

$\mathbf{F}$

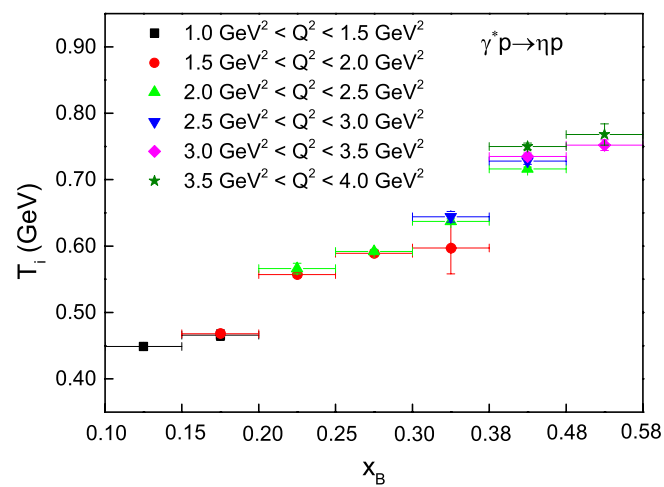

H

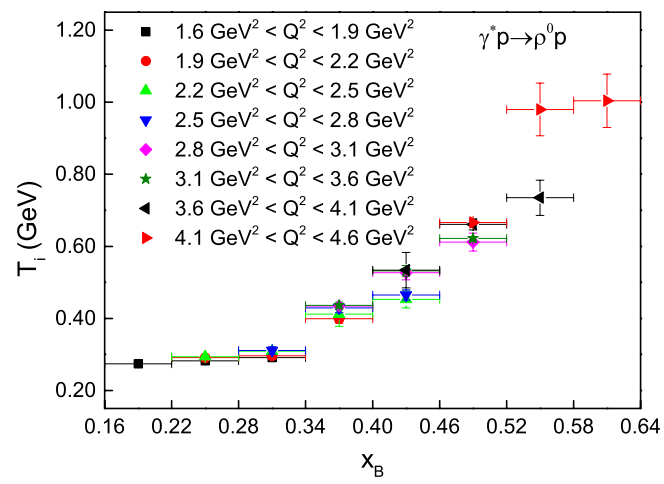

FIGURE 6 | The dependences of $\left\langle p_{T}\right\rangle$ (A,C,E,G) and $T_{i}(\mathbf{B}, \mathbf{D}, \mathbf{F}, \mathbf{H})$ on $x_{B}$ in $\gamma^{*} p$ collisions with emitted channels $\pi^{0} p(\mathbf{A}, \mathbf{B}), \pi^{+} n(\mathbf{C}, \mathbf{D}), \eta p(\mathbf{E}, \mathbf{F})$, and $\rho^{0} p(\mathbf{G}, \mathbf{H})$. Different symbols represent the results for different $Q^{2}$. 
One can see that the flow effect contributes largely to $\left\langle p_{T}\right\rangle$. It is expected that the contribution of flow effect increases with the increase of evolution time, if $\left\langle p_{T}\right\rangle$ is fixed from the initial- to final-states.

From Tables 1, 2, we note that the values of $n_{s}$ are 3-5 for different channels. As the number of participant partons, $n_{s}$ is constrained to be integer with uncertainty of 0 . For a given channel, $n_{s}$ is independent of $Q^{2}$ and $x_{B}$ in most cases. The channel independent $n_{s}$ renders that the number of participant partons is not too small or big. The number of struck parton(s) is usually regarded as 1 or 2 , which is very small. The struck parton(s) and the partons around the struck parton(s) are participant partons. The partons far away from the struck parton(s) are remainder or spectator partons.

Before summary and conclusion, we would like to point out that the discussion about the temperature and flow in this paper is applicable. Although the multiplicity in $e p$ collisions at a few $\mathrm{GeV}$ is very limited and the final particles are in a state far from thermal equilibrium, we may use the grand canonical ensemble for lots of events in which the number of total particles is very large and the whole system is in a homogeneous and equilibrium state. Therefore, the temperature used in this paper is comparable to the freeze-out temperatures used in nucleus-nucleus collisions. Of course, we may also regarded the temperature used here as a fitting parameter if necessary.

The initial-temperature $T_{i}$ is extracted from the root-meansquare of $p_{T}$, which is independent of model, though the relation between $T_{i}$ and $\sqrt{\left\langle p_{T}^{2}\right\rangle}$ is from the color string percolation method [43-46]. As deep inelastic scattering, ep collisions are head-on collisions, and may be harder than nucleus-nucleus collisions at similar energy per nucleon due to the fact that some non-head-on nucleon-nucleon collisions exist in the later. As a hybrid state of head-on and non-head-on nucleonnucleon collisions, nucleus-nucleus collisions may be weaker than head-on ep collisions. In addition, cold spectator nuclear effect also causes the temperature in nucleus-nucleus collisions to reduce. This renders that $T_{i}$ obtained in this paper is higher than that in nucleus-nucleus collisions.

It should be emphasized that the parameter $T_{i}$ reflects the violent degree of collisions. To our knowledge, other groups and other studies where $T_{i}$ is extracted for hadronic collisions is not available at present, though $T_{i}$ for nucleus-nucleus collisions is available. In terms of $T_{i}$, Erlang distribution, and Monte Carlo calculation, the present work has proposed an alternative method to describe light meson electroproduction data obtained with the JLab-CLAS facility. Typically those data are interpreted in terms of handbag diagram within the formalism of generalized parton distributions, whereas here statistical methods, that were developed for high-energy nucleus-nucleus collisions, are applied. At least, the present work has significance in the application of statistical methods.

\section{SUMMARY AND CONCLUSION}

In summary, the squared momentum transfer spectra of $\pi^{0}, \pi^{+}, \eta$, and $\rho^{0}$ produced in $\gamma^{*} p \rightarrow$ meson + nucleon process have been fitted by the calculated results with the Erlang distribution which is obtained from the multi-source thermal model and used to describe the transverse momentum spectra of emitted particles. The squared momentum transfer undergoes from the incident $\gamma^{*}$ to emitted meson, and also equivalently from the target proton to emitted nucleon. The model results are in agreement with the experimental data measured by the CLAS Collaboration. The values of the related parameters are extracted in the fitting process. The squared photon virtuality $Q^{2}$ and Bjorken variable $x_{B}$ dependent parameters are obtained.

With increasing of $Q^{2}$, the quantities $\left\langle p_{T}\right\rangle$ and $T_{i}$ increase generally. $Q^{2}$ is defined as absolute value of the squared mass of the virtual photon that is exchanged between the electron and the target proton, and it effectively represents the transverse size of the probe. $Q^{2}$ also reflects the hard scale of collisions. A harder scale results in a higher excitation degree of the system, and a larger $\left\langle p_{T}\right\rangle$ and $T_{i}$. At harder scale (larger $Q^{2}$ ), the degree of equilibrium decreases because of more disturbance to the equilibrated residual partons in target particle, though the system is at the state of high degree of excitation.

Similar to the tendency of $Q^{2}$, with an increase of $x_{B}$, the quantities $\left\langle p_{T}\right\rangle$ and $T_{i}$ increase. In the considered $\gamma^{*} p \rightarrow$ meson + nucleon process, $x_{B}$ represents the longitudinal momentum fraction transferred to the struck parton. The larger $x_{B}$ means the larger longitudinal momentum transfer to the system. It is natural that $\left\langle p_{T}\right\rangle$ and $T_{i}$ are larger at larger $x_{B}$. In addition, because $x_{B} \propto Q$, one may argue that $x_{B}$ also represents the hard scale of collisions. Indeed, it is understandable that larger $\left\langle p_{T}\right\rangle$ and $T_{i}$ appear at higher $x_{B}$.

\section{DATA AVAILABILITY STATEMENT}

The original contributions presented in the study are included in the article, further inquiries can be directed to the corresponding authors.

\section{AUTHOR CONTRIBUTIONS}

All authors listed have made a substantial, direct, and intellectual contribution to the work and approved it for publication.

\section{FUNDING}

The work of QW and F-HL was supported by the National Natural Science Foundation of China under Grant Nos 12047571, 11575103, and 11947418, the Scientific and Technological Innovation Programs of Higher Education Institutions in Shanxi (STIP) under Grant No. 201802017, the Shanxi Provincial Natural Science Foundation under Grant No. 201901D111043, and the Fund for Shanxi “1331 Project" Key Subjects Construction. The work of KKO was supported by the Ministry of Innovative Development of the Republic of Uzbekistan within the fundamental project No. F3-20200929146 on analysis of open data on heavy-ion collisions at RHIC and LHC. 


\section{REFERENCES}

1. Wang H, Chen J-H, Ma Y-G, Zhang S. Charm Hadron Azimuthal Angular Correlations in $\mathrm{Au}+\mathrm{Au}$ Collisions at $\sqrt{\mathrm{S}_{\mathrm{NN}}}=200 \mathrm{GeV}$ from Parton Scatterings. Nucl Sci Tech (2019) 30:185. doi:10.1007/s41365-019-0706-Z

2. Yan T-Z, Li S, Wang Y-N, Xie F, Yan T-F. Yield Ratios and Directed Flows of Light Particles from Proton-Rich Nuclei-Induced Collisions. Nucl Sci Tech (2019) 30:15. doi:10.1007/s41365-018-0534-6

3. Fisli M, Mebarki N. Top Quark Pair-Production in Noncommutative Standard Model. Adv High Energ Phys (2020) 2020:7279627. doi:10.1155/2020/7279627

4. He X-W, Wu F-M, Wei H-R, Hong B-H. Energy-Dependent Chemical Potentials of Light Hadrons and Quarks Based on Transverse Momentum Spectra and Yield Ratios of Negative to Positive Particles. Adv High Energ Phys (2020) 2020:1265090. doi:10.1155/2020/1265090

5. Waqas M, Li B-C. Kinetic Freeze-Out Temperature and Transverse Flow Velocity in Au-Au Collisions at RHIC-BES Energies. Adv High Energ Phys (2020) 2020:1787183. doi:10.1155/2020/1787183

6. Tang Z-B, Zha W-M, Zhang Y-F. An Experimental Review of Open Heavy Flavor and Quarkonium Production at RHIC. Nucl Sci Tech (2020) 31:81. doi:10.1007/s41365-020-00785-8

7. Shen C, Yan L. Recent Development of Hydrodynamic Modeling in Heavy-Ion Collisions. Nucl Sci Tech (2020) 31:122. doi:10.1007/s41365-020-00829-z

8. Yu H, Fang D-Q, Ma Y-G. Investigation of the Symmetry Energy of Nuclear Matter Using Isospin-dependent Quantum Molecular Dynamics. Nucl Sci Tech (2020) 31:61. doi:10.1007/s41365-020-00766-x

9. Bhaduri S, Bhaduri A, Ghosh D. Study of Di-muon Production Process in pp Collision in CMS Data from Symmetry Scaling Perspective. Adv High Energ Phys (2020) 2020:4510897. doi:10.1155/2020/4510897

10. Tawfik AN. Out-of-equilibrium Transverse Momentum Spectra of Pions at LHC Energies. Adv High Energ Phys (2019) 2019:4604608. doi:10.1155/2019/ 4604608

11. Nayak JK, Alam J-E., Sarkar S, Sinha B. Measuring Initial Temperature through a Photon to Dilepton Ratio in Heavy-Ion Collisions. J Phys G: Nucl Part Phys (2008) 35:104161. doi:10.1088/0954-3899/35/10/104161

12. Adare A, Afanasiev S, Aidala C, Ajitanand NN, Akiba Y, Al-Bataineh H, et al. Enhanced Production of Direct Photons in $\mathrm{Au}+\mathrm{Au}$ Collisions at $\sqrt{s}=200$ $\mathrm{GeV}$ and Implications for the Initial Temperature. Phys Rev Lett (2010) 104: 132301. doi:10.1103/PhysRevLett.104.132301

13. Csanád M, Májer I. Initial Temperature and EoS of Quark Matter via Direct Photons. Phys Part Nuclei Lett (2011) 8:1013-5. doi:10.1134/S1547477111090147

14. Csanád M, Májer I. Equation of State and Initial Temperature of Quark Gluon Plasma at RHIC. Cent Eur J Phys (2012) 10:850. doi:10.2478/s11534-0120060-9

15. Soltz RA, Garishvili I, Cheng M, Abelev B, Glenn A, Newby J, et al. Constraining the Initial Temperature and Shear Viscosity in a Hybrid Hydrodynamic Model of $\sqrt{s}=200 \mathrm{GeV} \mathrm{Au}+\mathrm{Au}$ Collisions Using Pion Spectra, Elliptic Flow, and Femtoscopic Radii. Phys Rev C (2013) 87: 044901. doi:10.1103/PhysRevC.87.044901

16. Waqas M, Liu F-H. Initial, Effective, and Kinetic Freeze-Out Temperatures from Transverse Momentum Spectra in High-Energy Proton(Deuteron)Nucleus and Nucleus-Nucleus Collisions. Eur Phys J Plus (2020) 135:147. doi:10.1140/epjp/s13360-020-00213-1

17. Cleymans J, Paradza MW. Tsallis Statistics in High Energy Physics: Chemical and thermal Freeze-Outs. Physics (2020) 2:654-64. doi:10.3390/physics 2040038

18. Li L-L, Liu F-H. Kinetic Freeze-Out Properties from Transverse Momentum Spectra of Pions in High Energy Proton-Proton Collisions. Physics (2020) 2: 277-308. doi:10.3390/physics2020015

19. Wang Q, Liu F-H, Olimov KK. Initial- and Final-State Temperatures of Emission Source from Differential Cross-Section in Squared Momentum Transfer in High-Energy Collisions. Adv High Energ Phys (2021) 2021: 6677885. doi:10.1155/2021/6677885

20. Liu F-H, Li J-S. Isotopic Production Cross Section of Fragments in ${ }^{56} \mathrm{Fe}+\mathrm{p}$ and ${ }^{136} \mathrm{Xe}\left({ }^{124} \mathrm{Xe}\right)+\mathrm{Pb}$ reactions over an Energy Range from $300 \mathrm{~A}$ to $1500 \mathrm{~A} \mathrm{MeV}$. Phys Rev C (2008) 78:044602. doi:10.1103/PhysRevC.78.044602

21. Liu F-H. Unified Description of Multiplicity Distributions of Final-State Particles Produced in Collisions at High Energies. Nucl Phys A (2008) 810: 159-72. doi:10.1016/j.nuclphysa.2008.06.014
22. Liu F-H, Gao Y-Q, Tian T, Li B-C. Unified Description of Transverse Momentum Spectrums Contributed by Soft and Hard Processes in High-Energy Nuclear Collisions. Eur Phys J A (2014) 50:94. doi:10.1140/epja/i2014-14094-9

23. Tsallis C. Possible Generalization of Boltzmann-Gibbs Statistics. J Stat Phys (1988) 52:479-87. doi:10.1007/BF01016429

24. Abelev BI, Adams J, Aggarwal MM, Ahammed Z, Amonett J, Anderson BD, et al. Strange Particle Production in $p+p$ Collisions at $\sqrt{s}=200 \mathrm{GeV}$. Phys Rev C (2007) 75:064901. doi:10.1103/PhysRevC.75.064901

25. Hagedorn R. Multiplicities, $\mathrm{p}_{\mathrm{T}}$ Distributions and the Expected Hadron $\rightarrow$ Quark-Gluon Phase Transition. Riv Nuovo Cim (1983) 6(10):1-50. doi:10.1007/BF02740917

26. Zhang NS. Particle Physics, Vol. I. Beijing, China: Science Press (1986). p. 116.

27. Sarkisyan EKG, Sakharov AS. Multihadron Production Features in Different Reactions. AIP Conf Proc (2006) 828:35. doi:10.1063/1.2197392

28. Sarkisyan EKG, Sakharov AS. Relating Multihadron Production in Hadronic and Nuclear Collisions. Eur Phys J C (2010) 70:533-41. doi:10.1140/epjc/ s10052-010-1493-1

29. Mishra AN, Sahoo R, Sarkisyan EKG, Sakharov AS. Effective-Energy Budget in Multiparticle Production in Nuclear Collisions. Eur Phys J C (2014) 74:3147. doi:10.1140/epjc/s10052-014-3147-1

30. Sarkisyan EKG, Mishra AN, Sahoo R, Sakharov AS. Multihadron Production Dynamics Exploring the Energy Balance in Hadronic and Nuclear Collisions. Phys Rev D (2016) 93:054046. doi:10.1103/PhysRevD.93.054046

31. Sarkisyan EKG, Mishra AN, Sahoo R, Sakharov AS. Centrality Dependence of Midrapidity Density from $\mathrm{GeV}$ to $\mathrm{TeV}$ Heavy-Ion Collisions in the Effective-Energy Universality Picture of Hadroproduction. Phys Rev D (2016) 94:011501. doi:10.1103/PhysRevD.94.011501

32. Sarkisyan-Grinbaum EK, Nath Mishra A, Sahoo R, Sakharov AS. EffectiveEnergy Universality Approach Describing Total Multiplicity Centrality Dependence in Heavy-Ion Collisions. EPL (2019) 127:62001. doi:10.1209/ 0295-5075/127/62001

33. Mishra AN, Ortiz A, Paić G. Intriguing Similarities of High- $\mathrm{p}_{\mathrm{T}}$ Particle Production between pp and A-A Collisions. Phys Rev C (2019) 99:034911. doi:10.1103/PhysRevC.99.034911

34. Castorina P, Plumari S, Satz H. Universal Strangeness Production in Hadronic and Nuclear Collisions. Int J Mod Phys E (2016) 25:1650058. doi:10.1142/ S0218301316500580

35. Castorina P, Iorio A, Lanteri D, Satz H, Spousta M. Universality in High Energy Collisions of Small and Large Systems. In: Proceedings of the 40th International Conference on High Energy Physics - ICHEP2020; July 28 August 6, 2020; Prague, Czech Republic, 390 (2021). p. 537. virtual meeting. doi:10.22323/1.390.0537

36. Castorina P, Iorio A, Lanteri D, Satz H, Spousta M. Universality in Hadronic and Nuclear Collisions at High Energy. Phys Rev C (2020) 101:054902. doi:10.1103/PhysRevC.101.054902

37. Bedlinskiy I, Kubarovsky V, Niccolai S, Stoler P, Adhikari KP, Anderson MD, et al.CLAS Collaboration. Exclusive $\pi^{0}$ Electroproduction at $W>2 \mathrm{GeV}$ with CLAS. Phys Rev C (2014) 90:025205. doi:10.1103/PhysRevC.90.025205

38. Park K, Guidal M, Gothe RW, Laget JM, Garçon M, Adhikari KP, et al.CLAS Collaboration. Deep Exclusive $\pi^{+}$Electroproduction off the Proton at CLAS. Eur Phys J A (2013) 49:1. doi:10.1140/epja/i2013-13016-9

39. Bedlinskiy I, Kubarovsky V, Stoler P, Adhikari KP, Akbar Z, Pereira SA, et al. Exclusive $\eta$ Electroproduction at $W>2 \mathrm{GeV}$ with CLAS and Transversity Generalized Parton Distributions. Phys Rev C (2017) 95:035202. doi:10.1103/ PhysRevC.95.035202

40. Morrow SA, Guidal M, Garçon M, Laget JM, Smith ES, Adams G, et al. Exclusive $\rho^{0}$ Electroproduction on the Proton at CLAS. Eur Phys J A (2009) 39: 5. doi:10.1140/epja/i2008-10683-5

41. An CS, Saghai B. Sea-Quark Flavor Content of Octet Baryons and Intrinsic Five-Quark Fock States. Phys Rev C (2012) 85:055203. doi:10.1103/ PhysRevC.85.055203

42. An CS, Saghai B. Strangeness Magnetic Form Factor of the Proton in the Extended Chiral Quark Model. Phys Rev C (2013) 88:025206. doi:10.1103/ PhysRevC.88.025206

43. Gutay LJ, Hirsch AS, Scharenberg RP, Srivastava BK, Pajares C. DeConfinement in Small Systems: Clustering of Color Sources in High Multiplicity $\bar{p} p$ Collisions at $\sqrt{s}=1.8 \mathrm{TeV}$. Int J Mod Phys E (2015) 24: 1550101. doi:10.1142/S0218301315501013 
44. Scharenberg RP, Srivastava BK, Pajares C. Exploring the Initial Stage of High Multiplicity Proton-Proton Collisions by Determining the Initial Temperature of the Quark-Gluon Plasma. Phys Rev D (2019) 100:114040. doi:10.1103/ PhysRevD.100.114040

45. Sahoo P, De S, Tiwari SK, Sahoo R. Energy and Centrality Dependent Study of Deconfinement Phase Transition in a Color String Percolation Approach at RHIC Energies. Eur Phys J A (2018) 54:136. doi:10.1140/epja/ i2018-12571-9

46. Wang Q, Liu F-H. Excitation Function of Initial Temperature of Heavy Flavor Quarkonium Emission Source in High Energy Collisions. Adv High Energ Phys (2020) 2020:5031494. doi:10.1155/2020/5031494

47. Aaron FD, Martin MA, Alexa C, Andreev V, Antunovic B, Asmone A, et al. Diffractive Electroproduction of $\rho$ and $\phi$ Mesons at HERA. J High Energ Phys (2010) 2010(05):32. doi:10.1007/JHEP05(2010)032

48. Aktas A, Andreev V, Anthonis T, Antunovic B, Aplin S, Asmone A, et al. Elastic J/ $\psi$ Production at HERA. Eur Phys J C (2006) 46:585-603. doi:10.1140/ epjc/s2006-02519-5

49. Chekanov S, Derrick M, Magill S, Musgrave B, Nicholass D, Repond J, et al. Exclusive $\rho 0$ Production in Deep Inelastic Scattering at HERA. PMC Phys A (2007) 1:6. doi:10.1186/1754-0410-1-6

50. Derrick M, Krakauer D, Magill S, Mikunas D, Musgrave B, Okrasiński JR, et al. Measurement of Elastic $\omega$ Photoproduction at HERA ZEUS Collaboration. $Z$ Phys C (1997) 73:73. doi:10.1007/s00288005029710.1007/s002880050314

51. Chekanov S, Derrick M, Magill S, Miglioranzi S, Musgrave B, Repond J, et al. Exclusive Electroproduction of $\phi$ Mesons at HERA. Nucl Phys B (2005) 718:3. doi:10.1016/j.nuclphysb.2005.04.009

52. Chekanov S, Derrick M, Loizides JH, Magill S, Miglioranzi S, Musgrave B, et al. Exclusive Electroproduction of $J / \psi$ Mesons at HERA. Nucl Phys B (2004) 695:3-37. doi:10.1016/j.nuclphysb.2004.06.034
53. Barberis D, Binon FG, Close FE, Danielsen KM, Donskov SV, Earl BC, et al. A Coupled Channel Analysis of the Centrally Produced $\mathrm{K}^{+} \mathrm{K}^{-}$and $\pi^{+} \pi^{-}$Final States in pp Interactions at $450 \mathrm{GeV} / \mathrm{c}$. Phys Lett $B$ (1999) 462: 462-70. doi:10.1016/S0370-2693(99)00909-0

54. Barberis D, Beusch W, Binon FG, Blick AM, Close FE, Danielsen KM, et al. A Measurement of the Branching Fractions of the $f_{1}(1285)$ and $f_{1}(1420)$ Produced in central pp Interactions at $450 \mathrm{GeV} / \mathrm{c}$. Phys Lett $B$ (1998) 440: 225-32. doi:10.1016/S0370-2693(98)01264-7

55. Giacalone G. A Matter of Shape: Seeing the Deformation of Atomic Nuclei at High-Energy Colliders. arXiv:2101.00168. France: Université Paris-Saclay (2021). p. 50. Ph.D. Thesis.

Conflict of Interest: The authors declare that the research was conducted in the absence of any commercial or financial relationships that could be construed as a potential conflict of interest.

Publisher's Note: All claims expressed in this article are solely those of the authors and do not necessarily represent those of their affiliated organizations, or those of the publisher, the editors and the reviewers. Any product that may be evaluated in this article, or claim that may be made by its manufacturer, is not guaranteed or endorsed by the publisher.

Copyright (c) 2021 Wang, Liu and Olimov. This is an open-access article distributed under the terms of the Creative Commons Attribution License (CC BY). The use, distribution or reproduction in other forums is permitted, provided the original author(s) and the copyright owner(s) are credited and that the original publication in this journal is cited, in accordance with accepted academic practice. No use, distribution or reproduction is permitted which does not comply with these terms. 